\title{
Accelerated Wound Healing in Minipigs by On-Site Production and Delivery of CXCL12 by Transformed Lactic Acid Bacteria
}

\author{
Emelie Öhnstedt ${ }^{1,2}$, Hava Lofton Tomenius ${ }^{1,2}$, Peter Frank ${ }^{2}$, Stefan Roos ${ }^{3}$, Evelina Vågesjö ${ }^{1,2} \mathbb{D}$ \\ and Mia Phillipson 1,4,*
}

check for

updates

Citation: Öhnstedt, E.; Lofton

Tomenius, H.; Frank, P.; Roos, S.;

Vågesjö, E.; Phillipson, M.

Accelerated Wound Healing in

Minipigs by On-Site Production and

Delivery of CXCL12 by Transformed

Lactic Acid Bacteria. Pharmaceutics

2022, 14, 229. https://doi.org/

$10.3390 /$ pharmaceutics14020229

Academic Editors: Rossella Laurano and Monica Boffito

Received: 1 December 2021

Accepted: 12 January 2022

Published: 19 January 2022

Publisher's Note: MDPI stays neutral with regard to jurisdictional claims in published maps and institutional affiliations.

Copyright: (c) 2022 by the authors. Licensee MDPI, Basel, Switzerland. This article is an open access article distributed under the terms and conditions of the Creative Commons Attribution (CC BY) license (https:// creativecommons.org/licenses/by/ $4.0 /)$.
1 Department of Medical Cell Biology, Uppsala University, 75123 Uppsala, Sweden; emelie.ohnstedt@mcb.uu.se (E.Ö.); hava.lofton-tomenius@mcb.uu.se (H.L.T.); evelina.vagesjo@ilyapharma.se (E.V.)

2 Ilya Pharma AB, Dag Hammarskjölds Väg, 75237 Uppsala, Sweden; peter.frank@ilyapharma.se

3 Department of Molecular Sciences, Swedish University of Agricultural Sciences, 75651 Uppsala, Sweden; Stefan.Roos@slu.se

4 The Science for Life Laboratory, Uppsala University, 75237 Uppsala, Sweden

* Correspondence: mia.phillipson@mcb.uu.se

\begin{abstract}
Non-healing wounds are a growing medical problem and result in considerable suffering The lack of pharmaceutical treatment options reflects the multistep wound healing process, and the complexity of both translation and assessment of treatment efficacy. We previously demonstrated accelerated healing of full-thickness wounds in mice following topical application of the probiotic bacteria Limosilactobacillus reuteri R2LC transformed to express CXCL12. In this study, safety and biological effects of a freeze-dried formulation of CXCL12-producing L. reuteri (ILP100) were investigated in induced full-thickness wounds in minipigs, and different wound healing evaluation methods (macroscopic, planimetry, 2D-photographs, 3D-scanning, ultrasound) were compared. We found that treatment with ILP100 was safe and accelerated healing, as granulation tissue filled wound cavities 1 day faster in treated compared to untreated/placebo-treated wounds. Furthermore, evaluation using planimetry resulted in 1.5 days faster healing than using 2D photographs of the same wounds, whereas the areas measured using 2D photographs were smaller compared to those obtained from 3D scans accounting for surface curvatures, whereas ultrasound imaging enabled detailed detection of thin epithelial layers. In conclusion, topical administration of the drug candidate ILP100 warrants further clinical development as it was proven to be safe and to accelerate healing using different evaluation methods in minipigs.
\end{abstract}

Keywords: topical; Limosilactobacillus reuteri; wound measurement; active wound care; chemokines; three-dimensional imaging

\section{Introduction}

The skin serves as an important barrier to the environment, and wounding of the skin rapidly initiates a healing process. How quickly the wound closes depends on wound size and location, and is also influenced by age, underlying medical conditions, nutritional status, and medications [1-3]. Occasionally, the healing process is impaired and the wound is referred to as non-healing if it has failed to heal within 3 months. Non-healing wounds are a growing medical problem associated with aging populations and the prevalence of metabolic diseases [2]. In addition to causing discomfort and pain, such wounds increase the risk of amputation due to infections and result in associated care costs that can account for over $3 \%$ of the healthcare budget in industrialized countries $[4,5]$. There are currently very limited options for active treatment, i.e., treatments that accelerate wound healing. Instead, available therapies aim to protect the wound and create a moist wound environment favorable for healing [6]. Effective drugs that actively promote healing of these complicated 
wounds would not only alleviate suffering and conserve healthcare resources, but also reduce the use of antibiotics.

The complex process of wound healing can be divided into four consecutive and overlapping phases: hemostasis, inflammation, proliferation, and remodeling. During hemostasis, the bleeding is restricted by vasoconstriction followed by clot formation. Immune cells accumulate at the wound site during the inflammation phase and are important for fighting pathogens. It has become increasingly evident that the immune cells at the wound site also play a crucial part in orchestrating wound healing during the proliferative and remodeling phases during which granulation occurs, the wound is re-epithelialized, and the scar is formed.

The development of treatments to accelerate wound healing is associated with many challenges, which explains the limited range of available options. For instance, topical administration of drug candidates is limited by the proteolytic microenvironment of the wounds, which greatly reduces bioavailability [7]. We recently developed a means to circumvent this issue by transforming a strain of the probiotic bacteria Limosilactobacillus reuteri $\mathrm{R} 2 \mathrm{LC}$ (L. reuteri $\mathrm{R} 2 \mathrm{LC}$, previously known as Lactobacillus reuteri $\mathrm{R} 2 \mathrm{LC}$ ) to express murine or human CXCL12, which allows continuous expression of the protein at the wound site while inhibiting degradation of the chemokine [8]. Topical application of this genetically engineered $L$. reuteri R2LC was demonstrated to accelerate healing of full-thickness wounds in otherwise healthy or diabetic mice, and mice with peripheral hind limb ischemia, as well as to improve re-epithelialization using an ex vivo model of human skin disks [8]. This effect was proven to be macrophage-dependent, and both macrophage numbers and their transforming growth factor $\beta$ (TGF- $\beta$ ) production increased by CXCL12-producing $L$. reuteri R2LC treatment, which ultimately resulted in increased proliferation of keratinocytes and accelerated wound healing [8].

Another challenge for drug development to increase wound healing is the translation from the most readily available animal models in rodents to the clinic. For instance, the healing of mouse skin wounds differs in many aspects from that in humans. The skin of rodents is loosely attached to the subcutaneous connective tissue and contains a thin muscle layer (panniculus carnosus), which enables the wound to contract by bringing the wound edges close together [9-11]. In contrast, wound closure in pigs and humans solely depends on the formation of granulation tissue and re-epithelialization, as wound contraction does not occur since the skin is firmly attached to the underlying connective tissue and lacks the required muscle layer [9-11]. In addition, non-healing wounds form a heterogenous group as they are the result of several underlying and complex conditions, making them impossible to fully replicate preclinically. The available non-healing wound models usually consist of wounds induced in an animal model of a primary condition associated with non-healing wounds, such as ischemia and diabetes. However, non-healing wounds have a more multifaceted pathophysiology and also depend on factors such as age and bacterial load $[2,6,12]$.

Translation of preclinical protocols is moreover hampered by the current primary endpoints of clinical trials, as these do not reflect the wound healing process. The approved primary endpoints for clinical trials for chronic cutaneous ulcers and burn wounds, according to the FDA guidelines from 2006, are complete healing, facilitation of surgical closing, or reduced scarring $[13,14]$. The narrowness of these endpoints has been questioned by both investigators and clinicians, as they do not report the progression or quality of the wound healing process. Furthermore, additional information collected during monitoring of the healing process forms an untapped resource, which complements the conventional documentation of wound appearance and area [3]. An ongoing collaborative effort with the FDA therefore aims to identify new primary endpoints with improved clinical relevance and patient value [15], and has so far recognized reduced pain, fewer infections, and increased physical function/ambulation as the three most important parameters [16]. Based on these results and the work of the Wound Healing Endpoints Committee led by Driver et al., the FDA recently acknowledged five new endpoints [14]. 
The main objective of this paper was to increase the quality of wound healing evaluation by exploring and comparing classic as well as novel methods for the assessment of healing of induced, full-thickness wounds in minipigs. In parallel, the effect of the freezedried formulation of human CXCL12-producing L. reuteri R2LC, ILP100 a new-in-class drug candidate, on wound healing was evaluated in two separate cohorts of minipigs.

\section{Materials and Methods}

\subsection{Study Design}

Two separate studies were performed as part of the GLP toxicity program for ILP100; herein referred to as Cohort A and B, conducted two years apart. The primary objective of both studies was to assess safety and toxicity for regulatory compliance. The two studies also contained a number of complimentary technical and analytical exploratory endpoints which are reported herein.

\subsection{Animals}

Cohort A was performed in 18 male, and Cohort B in 15 female Göttingen SPF minipigs (Ellegaard Göttingen Minipigs A/S, Dalmose, Denmark) at CitoxLabs (Ejby, Denmark). At cohort inclusion, the pigs were randomized to the different treatment groups, weighed 19$25 \mathrm{~kg}$, and were between 7 to 11 months old. All experiments were approved by The Danish Veterinary and Food administration Council (Ethical permit number; 2015-15-0201-00713).

\subsection{Limosilactobacillus reuteri R2LC Encoding Human CXCL12}

A strain of probiotic bacteria Limosilactobacillus reuteri R2LC (L. reuteri R2LC) genetically engineered to encode human CXCL12 1 alpha has been designed as reported elsewhere [8], and developed in a freeze-dried formulation as the drug candidate ILP100. In brief, the sequences encoding the human chemokine CXCL12 1 alpha were inserted into an expression vector, after which the constructs were transformed into L. reuteri R2LC. The CXCL12 expression is induced by the addition of an inducing peptide, SppIP, resulting in the transformed L. reuteri R2LC expressing human CXCL12 following activation (for details [8]).

\subsection{Wound Induction}

The animals were anesthetized on day 1 . The anesthesia protocols differed slightly between Cohort A and Cohort B: both cohorts received, an intramuscular injection a mixture of Zoletil $50^{\circledR}$ Vet, (125 mg tiletamine and $125 \mathrm{mg}$ zolazepam), $20 \mathrm{mg}$ xylazine $/ \mathrm{mL}(6.25 \mathrm{~mL}$ ), $100 \mathrm{mg}$ ketamine $/ \mathrm{mL}(1.25 \mathrm{~mL})$ and $10 \mathrm{mg}$ butorphanol $/ \mathrm{mL}(2.5 \mathrm{~mL})$. In addition to these injections, the minipigs in Cohort $\mathrm{A}$ received an intramuscular injection of $40 \mathrm{mg}$ azaperone $(1 \mathrm{~mL} / 20 \mathrm{~kg})$ and $1 \mathrm{mg}$ atropine $/ \mathrm{mL}(0.05 \mathrm{~mL} / \mathrm{kg})$. The minipigs in Cohort B did not receive the azaperone/atropine injection and the anesthesia was instead maintained by isoflurane.

Prior to wound induction, the back of the animal was shaved, washed, and disinfected. Two to three circular full-thickness wounds $\left(20 \mathrm{~mm}\right.$ diameter, area $\left.3.14 \mathrm{~cm}^{2}\right)$ were induced on each side of the spine on the back of each animal.

For prevention of post-surgical pain due to wound induction, the animals were treated with fentanyl dermal patches (Cohort A $75 \mu \mathrm{g} / \mathrm{h}$, Cohort B $50 \mu \mathrm{g} / \mathrm{h}$ ) and a daily intramuscular injection meloxicam (Cohort A $20 \mathrm{mg} / \mathrm{mL}, 0.02 \mathrm{~mL} / \mathrm{kg}$, Cohort B $5 \mathrm{mg} / \mathrm{mL}$, $0.08 \mathrm{~mL} / \mathrm{kg}$ ) for up to $72 \mathrm{~h}$.

\subsection{Blood Sampling}

In Cohort $\mathrm{A}$, blood samples were drawn from the jugular vein before the start of treatment, on day 4 and on the day of the euthanization. In Cohort B, the blood samples were drawn from the jugular vein or the ear vein catheter before the start of treatment, on day 3 and on the day of the euthanization. All animals were fasted overnight with 
free access to water before blood sampling. For both cohorts, conventional panels for hematology and clinical chemistry were assessed.

\subsection{Urinalysis}

Before the start of treatment and before the termination of treatment, urine was collected from all animals. Urine samples were collected overnight from the stainless-steel tray under the stall and were assessed on conventional panels for urinalysis.

\subsection{Histopathology}

At euthanization, necropsy samples from all animal tissues were taken for histological processing (Table A1). The specimens were embedded in paraffin and cut at a nominal thickness of approximately $5 \mu \mathrm{m}$, stained with haematoxylin and eosin, and examined under a light microscope. Histological alterations were graded on a 5-level scale (minimal, mild, moderate, marked, and severe) by a pathologist, and selected slides were examined by a peer-reviewing pathologist.

\subsection{Wound Treatment}

The wounds were treated and the dressings were changed on days $1,2,3,5,7,9,11$, $13,15,17,19,21,25$, and 28 following wound induction (Figure 1). Cohort A comprised three subsets in which the wounds: (i) did not receive any treatment, (ii) were treated with wild type $L$. reuteri $\mathrm{R} 2 \mathrm{LC}$ ( $500 \mu \mathrm{L}, 2.5 \times 10^{9} \mathrm{CFU} /$ wound), (iii) were treated with ILP100 $\left(100 \mu \mathrm{L}, 7 \times 10^{9} \mathrm{CFU} /\right.$ wound). In Cohort B, the wounds were treated either with (i) placebo (500 $\mu \mathrm{L})$ or (ii) ILP100 $\left(500 \mu \mathrm{L}, 2.5 \times 10^{9} \mathrm{CFU} /\right.$ wound). Before treatment of the wounds, the freeze-dried formulations were reconstituted in buffer and activated with abundant amounts of SppIP (100 to $1000 \mathrm{ng} / \mathrm{mL}$ ).

\subsection{Wound Evaluation}

The wounds were macroscopically evaluated and photographed in a standardized manner at day 1 (only photo), 2, 3, 5, 7, 9, 11, 13, 15, 17, 19, 21, and 28 in Cohort A, and at day 1 (only photo), 3, 7, 9, 11, 13, 15, 21, 25 and 28 in Cohort B (Figure 1). The macroscopic evaluation performed on-site included scoring of granulation, presence of hypergranulation, wound edge inflammation, surrounding skin inflammation, hemorrhaging, and exudation. The scoring ranged from 0 -not present, 1-minimal, 2-slight, 3-moderate, and 4marked.

\subsubsection{Two-Dimensional Photographs of Wounds}

Two-dimensional (2D) photographs were taken in a standardized manner with a flash using the same camera and at a fixed distance with a $5.5 \mathrm{~cm} \times 5.5 \mathrm{~cm}$ frame placed around the wound. From the photographs, area measurements were performed using ImageJ2 software (National Institutes of Health, Bethesda, MD, USA) where the frame in the photos served as the scale.

\subsubsection{Planimetric Assessments of Wounds}

In Cohort $\mathrm{A}$, the wounds were measured using planimetry days 2, 3, 5, 7, 9, 11, 13, 15, $17,19,21$, and 28. For the planimetry assessment, a sterile transparent sheet was placed on top of the wound, on which both the area of the wound and the newly formed epithelia were outlined. In the wound, the area covered with granulation tissue was outlined and the remaining wound area was marked as unspecific tissue. The sheets were later analyzed using PictZar Pro (7.5.1) (Advanced Planimetric Services, Elmwood Park, NJ, USA). 


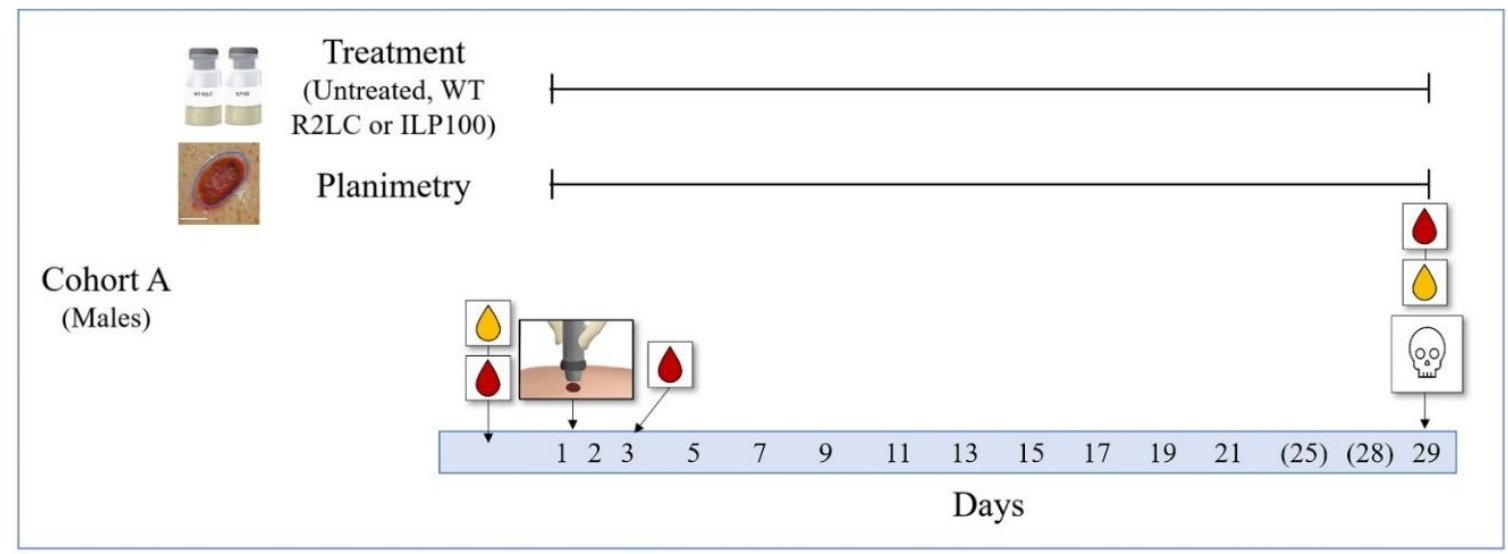

Both

2D photographs

Macroscopic

evaluation

Cohort B

(Females)

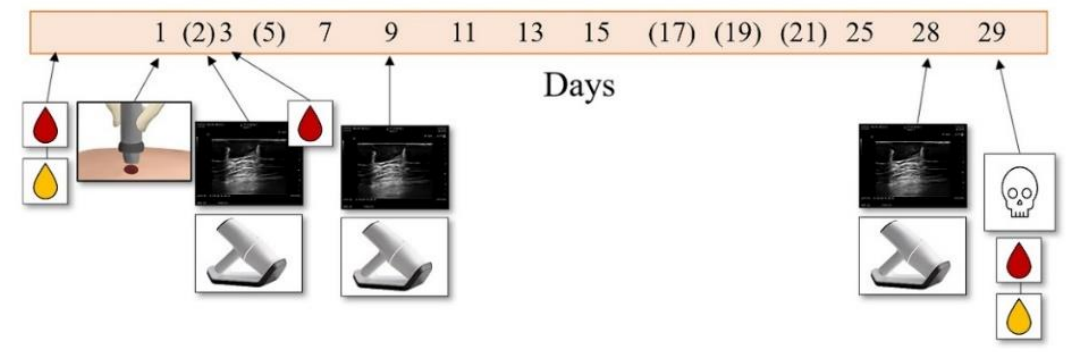

Treatment (Placebo or ILP100)

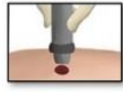

Wound induction

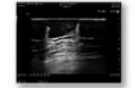

Ultrasound

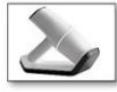

3D

scanning

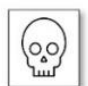

Euthanasia

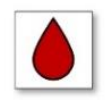

Blood sample

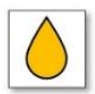

Urine

sample

Figure 1. Schematic illustration of the protocol design. The study consists of two cohorts: one in males (Cohort A, blue box) and one in females (Cohort B, orange box). In the illustration, the numbers in the filled blue (Cohort A) and orange (Cohort B) boxes indicate the days where 2D photographs, macroscopic evaluation, treatment, and planimetry (only Cohort A) were performed. No 2D photographs, macroscopic evaluation, or planimetry were performed on the days in parenthesis. Time points for wound induction, collection of blood (for hematology, clinical chemistry, plasma levels of CXCL12 and SppIP, and for CFU counts of ILP100), collection of urine samples, and for additional imaging (only Cohort B) with ultrasound and 3D scanning are indicated with arrowed symbols.

\subsubsection{Three-Dimensional Scanning of Wounds}

In Cohort B, three-dimensional wound measurements were carried out using a stereoscopic optical system, a Cherry Imaging platform consisting of a hand-held 3D scanner, and TraceTM version 5 software (Cherry Imaging, Yokneam, Israel, 2019). The 3D scanner acquires thousands of images with a speed of 15 frames/second at a resolution of $100 \mu \mathrm{m}$, that are rendered into a 3D surface [17]. For each pig, three of the wounds were scanned on days 2,9 , and 28 . The wound margins were manually marked on the 3D surfaces created in the TraceTM software, and the program then calculated the area, volume, and depth of the 
wound. Wound depth was designated as the average of $10 \%$ of the measured spots with the deepest values.

\subsubsection{Ultrasound Imaging of Wounds}

In Cohort B, the three wounds that were 3D scanned were imaged using ultrasound on days 2, 9, and 28 following wound induction using Arietta V60 with linear probes L64, 5-18 MHz. The probe was placed in the same direction on all occasions for all wounds, in order to produce scans that visualized one transversal section of the entire wound. Scanning directly on the wound was only possible using saline flushed into the wound cavity (Days 2 and 9). On Day 28, the wounds were completely healed and epithelialized, and ultrasound gel was used instead of saline.

\subsection{Data Management and Statistics}

Some data points from 2D photographs (123 of 2058) and 3D scans (32 of 135) are missing due to poor image quality, precluding reliable analysis. In addition, ultrasound images from eleven minipigs on day 2 were lost due to software failure during data transfer. All four wounds from one minipig from Cohort A in the ILP100 treated group were excluded since they all became infected. All data are presented as mean \pm SEM and/or with individual dots for each wound. Each wound was treated as an independent variable in the statistical analysis. Comparisons between area measurements achieved with different methods on the same wounds were performed with a Wilcoxon signed rank test. For comparison with unpaired values, the Mann-Whitney or Kruskal-Wallis test with Dunn's multiple comparison test was used. Jonckheere's trend test was used to assess if there were a trend between ordinal independent variables and continuous variables. $p<0.05$ was considered significant. All statistical analyses were performed using GraphPad Prism 9.0.1. (GraphPad Software, San Diego, CA, USA, 2021)

\section{Results}

\subsection{Evaluation of Methods Assessing Wound Granulation, Re-Epithelialization and Area}

Wound healing was assessed in minipigs using consecutive measurements of areas and volumes of induced wounds, as well as of the formed scars. Areas of wounds and early scars were measured and analyzed by three approaches: 2D photographs with ImageJ2 software, planimetry with PictZar Pro software, and 3D scans with the TraceTM software (Figure 1). Wound diameters were also measured by 2D photographs, 3D scans, and ultrasounds. Wound and scar volumes were assessed by 3D scanning, and all wounds were evaluated macroscopically for assessment of granulation tissue at different time points following induction.

In Cohort A, wound areas were assessed using planimetry and 2D photographs according to standardized protocols. On days 2, 5, 9, 11, and 13 following wound induction, there were statistically significant differences between the measured mean wound areas (93-96 wounds per time point) using planimetry or 2D photographs. However, of these time points, only days 9 and 11 had area differences exceeding $0.1 \mathrm{~cm}^{2}$, and the planimetrymeasured areas were $0.36( \pm 0.05) \mathrm{cm}^{2}$ and $0.20( \pm 0.04) \mathrm{cm}^{2}$ smaller for the respective days compared to the areas measured from 2D photographs (Figure 2A,B). Epithelialization was first noted on day 9 (Figure 2C) and might account for the observed area differences, as it is more difficult to detect thin epithelial layers from the 2D photographs acquired with a flash compared to those assessed by planimetry. In fact, the time to $50 \%, 75 \%$, and $100 \%$ re-epithelialization differed depending on the method used, as the time to $100 \%$ re-epithelialization occurred on average 1.5 days later $(p \leq 0.0001)$ when assessed by 2D photographs (planimetry: $12.2 \pm 2.3$ days, 2D photographs $13.6 \pm 2.1$ days, Figure 2D). Similarly, the $75 \%$ and $50 \%$ re-epithelialization were reached 1.0 day $(p \leq 0.0001)$ and 0.2 day $(p=0.08)$ earlier, respectively, when assessed by planimetry compared to $2 \mathrm{D}$ photographs (Figure 2E,F). 
A

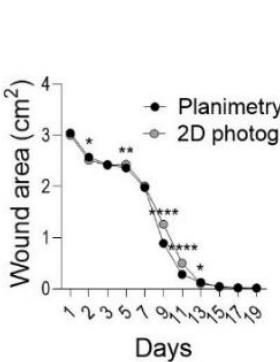

D
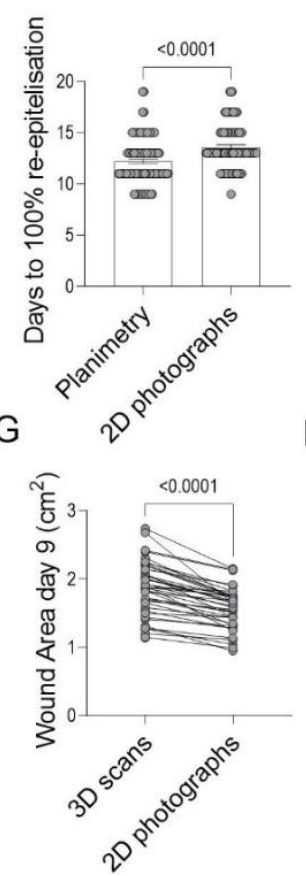

J

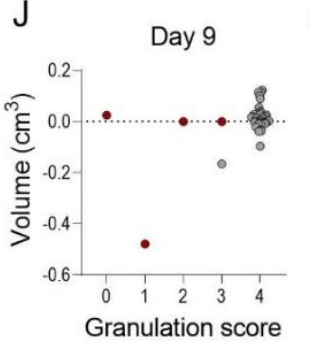

B

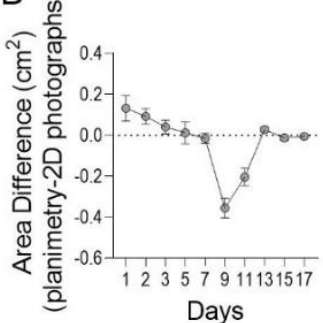

E

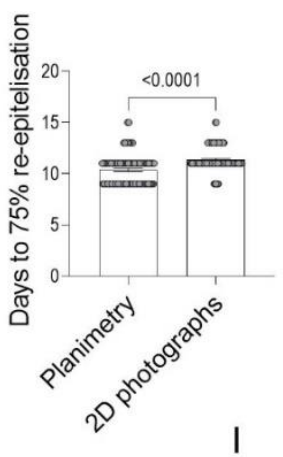

$\mathrm{H}$

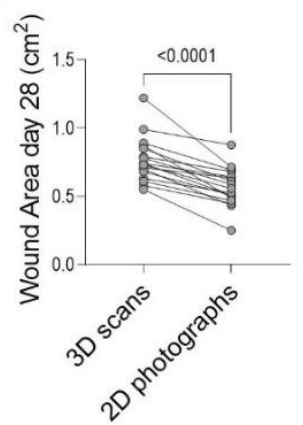

K

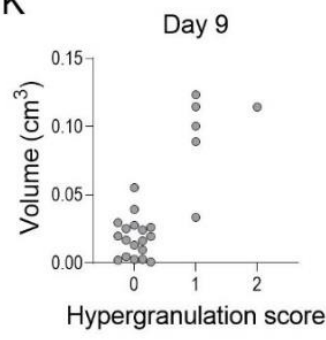

L
C

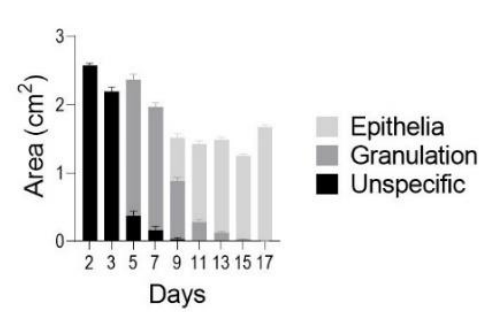

F

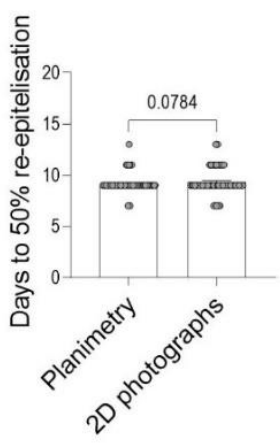

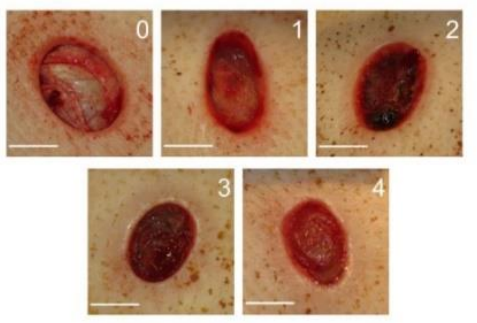

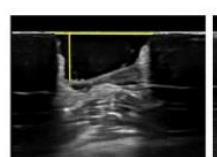

Day 2

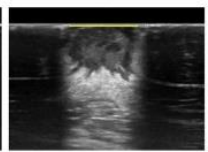

Day 9

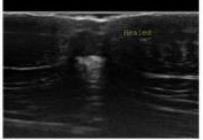

Day 28
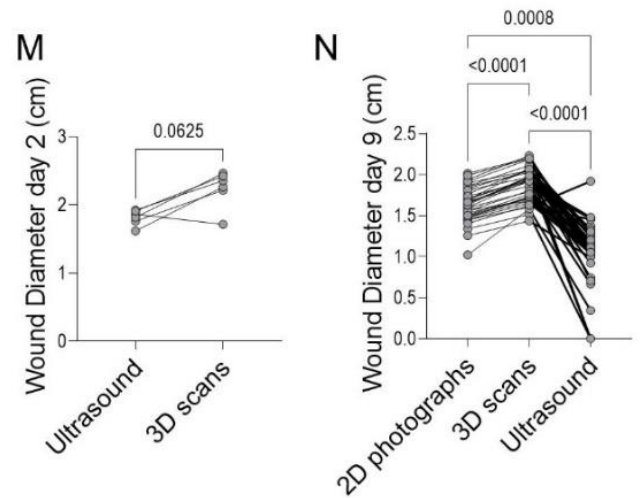

Figure 2. Comparisons of different wound assessment methods. Wound areas from Cohort A were measured by on-site planimetry (A) and from 2D photographs (B), $n=18, N=93-96)$, and the areas 
were compared to reveal method-dependent differences. From the planimetric data, the areas of newly formed epithelia, granulation tissue, and unspecific tissue for all wounds in Cohort A (C) were retrieved $(n=18, N=96)$. Furthermore, days to $100 \%(n=18, N=96), 75 \%(n=18, N=91)$, and $50 \%(n=18, N=91)$ re-epithelialisation of all wounds were assessed with planimetry and from $2 \mathrm{D}$ photographs (D-F) in Cohort A. In Cohort B, method-dependent differences between area measured with 3D scanning and from 2D photographs on day $9(\mathbf{G}), n=17, N=42)$, and day $28(\mathbf{H}), n=6$, $N=17$ ) were assessed. In addition, the formation of granulation tissue was assessed macroscopically by scoring from 0 to 4 (scale bar $1 \mathrm{~cm}$ ) (I). To assess correlations between macroscopic evaluations and volume measurements from the 3D scans at day 9, granulation score $(\mathrm{J})$ and hypergranulation score $(\mathbf{K})$ was plotted against volumes generated from the 3D scans of the respective wound ( $n=17$, $N=42$ ), where red dots indicate clot formation in the wound. On days 2,9 , and 28 , the wounds in Cohort B were imaged with ultrasound and representative images are shown (L), where the yellow lines delineate measured diameters and depth showing that the wounds are fully epithelialized and considered healed at $\mathrm{d} 28$. Method-dependent differences were assessed by comparing the diameter of each wound measured from images acquired by ultrasound or 3D scanning day 2 (M) and by ultra-ound, 3D scanning, or from 2D photographs on day $9(\mathbf{N})$. Statistic comparisons were made using Wilcoxon signed rank test $(\mathbf{A}, \mathbf{D}-\mathbf{F}, \mathbf{H}, \mathbf{I}, \mathbf{M})$, Jonckheere's trend test $(\mathbf{J})$ and Friedmans test with Dunn's multiple comparison test $(\mathbf{N})\left(^{*} p<0.05,{ }^{* *} p<0.01,{ }^{* * *} p<0.0001\right)$.

To allow measurements of the wound area, depth, and volume, as well as the height and volumes of scars, a technology with stereoscopic scanning generating 3D surface models was utilized in Cohort B. When the areas obtained from 3D scanning was compared to those obtained from the 2D photographs of the same wounds on day 9 and day 28, it became evident that smaller wound areas were detected by 2D photographs when compared to those from 3D scans (day 9: $16 \pm 8 \%$ smaller, $p \leq 0.0001$, day $28: 27 \pm 12 \%$ smaller, $p<0.0001$, Figure $2 \mathrm{G}, \mathrm{H})$.

The formation of granulation tissue in the wound cavity is a prerequisite for reepithelialization as it enables epithelial cell migration and wound closure (Figure 2I). The 3D scanning of wounds results in a negative volume corresponding to the wound cavity, while a positive volume depicts clot formation or hypergranulation, i.e., granulation tissue elevated beyond the level of the surrounding skin. The 3D scans were complemented with a macroscopic evaluation of the wounds before each treatment, and the wounds were scored (0-4) for the formation of granulation tissue, where higher scores indicate that a larger extent of the cavity is filled with granulation tissue. The granulation scoring and 3D volume measurements at day 9 were then plotted against each other to test for correlation. On day 9 , the majority of the wounds had a granulation score of 4 and a wound volume close to 0 (Figure 2J). If wounds with blood clots were excluded (red dots in graphs), the wound with the largest cavity was the one receiving a lower granulation score, as expected. Due to the fast formation of granulation tissue in the induced wounds, 3D scanning should be performed at earlier time points to evaluate if those results correlate with the macroscopic observations. Interestingly, all wounds that were scored as hypergranulating from the macroscopic evaluation also showed a positive volume when measured by the 3D scanning, and a significant trend ( $p=0.0001)$ towards higher hypergranulation scores with increasing volume was demonstrated using Jonckheere's trend test (Figure 2K).

Thus, wound healing can be evaluated using several parameters, and one method is not enough for assessing all aspects of the healing process. As demonstrated above, different methods might give different results when measuring the same parameters. Therefore, these differences should be taken into account when making informed decisions on which method to use in designing controlled studies assessing wound healing in animals and humans.

\subsection{Treatment with CXCL12-Producing L. reuteri R2LC Accelerates Wound Healing}

The biological effects of freeze-dried human CXCL12-producing L. reuteri R2LC (ILP100) following topical treatment of wounds were then assessed and compared to 
untreated wounds or wounds receiving placebo or wild-type L. reuteri R2LC (Figure 3A). Directly prior to treatment of the wounds with a dose of $10^{9}$ colony forming units (CFU) per wound, the freeze-dried bacterial formulation was reconstituted, and expression was induced by the addition of abundant amounts of SppIP $(100-1000 \mathrm{ng} / \mathrm{mL}$ supplied in the reconstitution buffer).

The biological effect of ILP100-treatment was demonstrated in Cohort A by accelerated re-epithelialization and increased formation of granulation tissue as compared to untreated wounds (Planimetry). Accelerated re-epithelialization by ILP100 was observed as reduced wound area and increased percentage of the wounds being re-epithelialized when compared to untreated wounds and wounds treated with wild-type L. reuteri R2LC (Planimetry, Figure 3B,C). In addition, larger areas of newly formed epithelia were demonstrated following ILP100-treatment on days 9 and 11, and the treated wounds became fully re-epithelialized 3 days earlier than untreated wounds (Planimetry, Figure 3D,E), even though no differences were detected for the time leading to $50 \%$ or $75 \%$ re-epithelialization of the wound area (Planimetry, Figure 3F,G). The accelerated re-epithelialization demonstrated by planimetry was also supported by assessments from the 2D photographs, as the ILP100-treated wounds were fully epithelized almost 2 days faster than the untreated wounds (12.6 \pm 0.4 days versus $14.4 \pm 1.4$ days, respectively, Figure A1). However, no differences were detected between treatments when absolute wound size, percent reepithelialized wound area, or time to $50 \%$ or $75 \%$ re-epithelialization were analyzed in the $2 \mathrm{D}$ photographs. The discrepancy observed for results obtained by planimetry and 2D photographs were in line with our evaluation that planimetry reported faster wound healing compared to 2D photographs (Figure 2D). In addition, the formation of granulation tissue was accelerated by the ILP100 treatment at day 5 , and the time to complete wound coverage of granulation tissue occurred 1.5 days earlier in the ILP100-treated wounds when compared to untreated wounds (Planimetry, Figure 3H,I). This observation was strengthened by the macroscopic evaluation, as ILP100-treated wounds received higher average scores for the assessment of granulation, starting at day 5 (Figure $3 \mathrm{~J}, \mathrm{~K}$ ).

In Cohort B, macroscopic evaluation again demonstrated increased granulation of ILP100-treated wounds at day 7 and day 9 when compared to placebo (Figure 4A,B), whereas no granulation scoring was performed at day 5 as part of the protocol. Planimetry was not performed in Cohort B, but data from 2D photographs revealed an increased portion of re-epithelialization of wounds following ILP100-treatment at day 7 and day 9 , resulting in the ILP100-treated wounds reaching 75\% and 50\% re-epithelialization area faster than the placebo-treated wounds (Figure A2B,D,E). No differences in wound area or time to complete re-epithelialization between treatments could, however, be detected in analyses from the 2D photographs (Figure A2A,C).

The wounds in Cohort $\mathrm{B}$ were also imaged by the 3D scanner on three occasions (day 2,9 , and 28 , Figure $4 \mathrm{C}$ ). In accordance with the observations from the $2 \mathrm{D}$ photographs, a reduced wound area was observed on day 9 for the ILP100-treated wounds using the 3D scans (Figure 4D). The 3D scans also revealed a reduced depth (calculated as the mean of the deepest $10 \%$ of measurements of the wound) in the ILP100-treated wounds compared to placebo (Figure 4E), even though no statistical differences in wound volumes were observed (Figure 4F). Early scarring was evaluated using the 3D scans at day 28, where no statistical differences could be observed between treatments, even though a trend of reduced scar area $(p=0.0923)$ and reduced scar height $(p=0.0975$, Figure 4G-I) were detected for the ILP100 treated wounds.

Taken together, topical ILP100 treatment to induced full-thickness wounds accelerated wound healing in both male and female minipigs. 
A
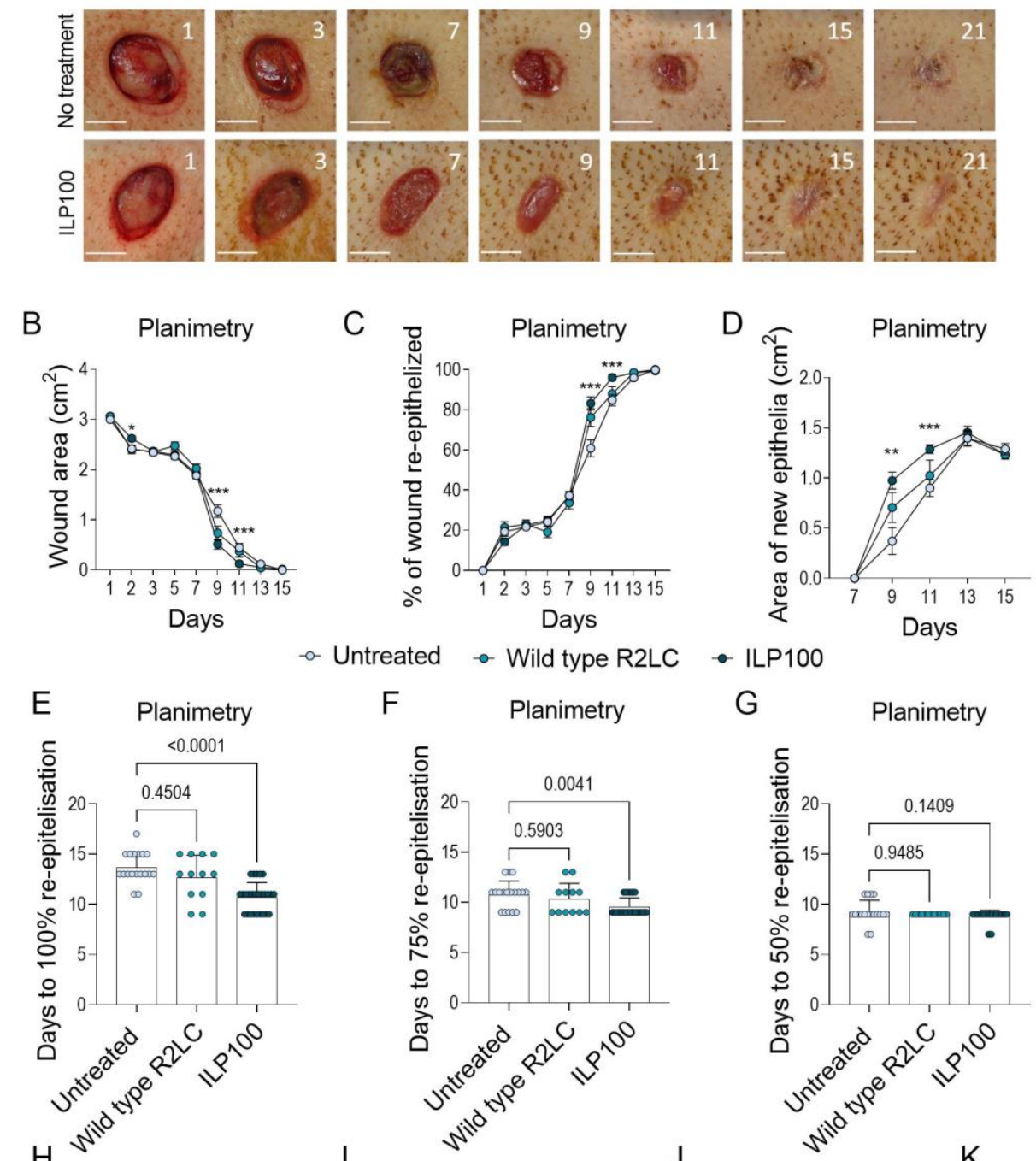

$\rightarrow$ Untreated $\rightarrow$ Wild type R2LC

G

F Planimetry

Planimetry
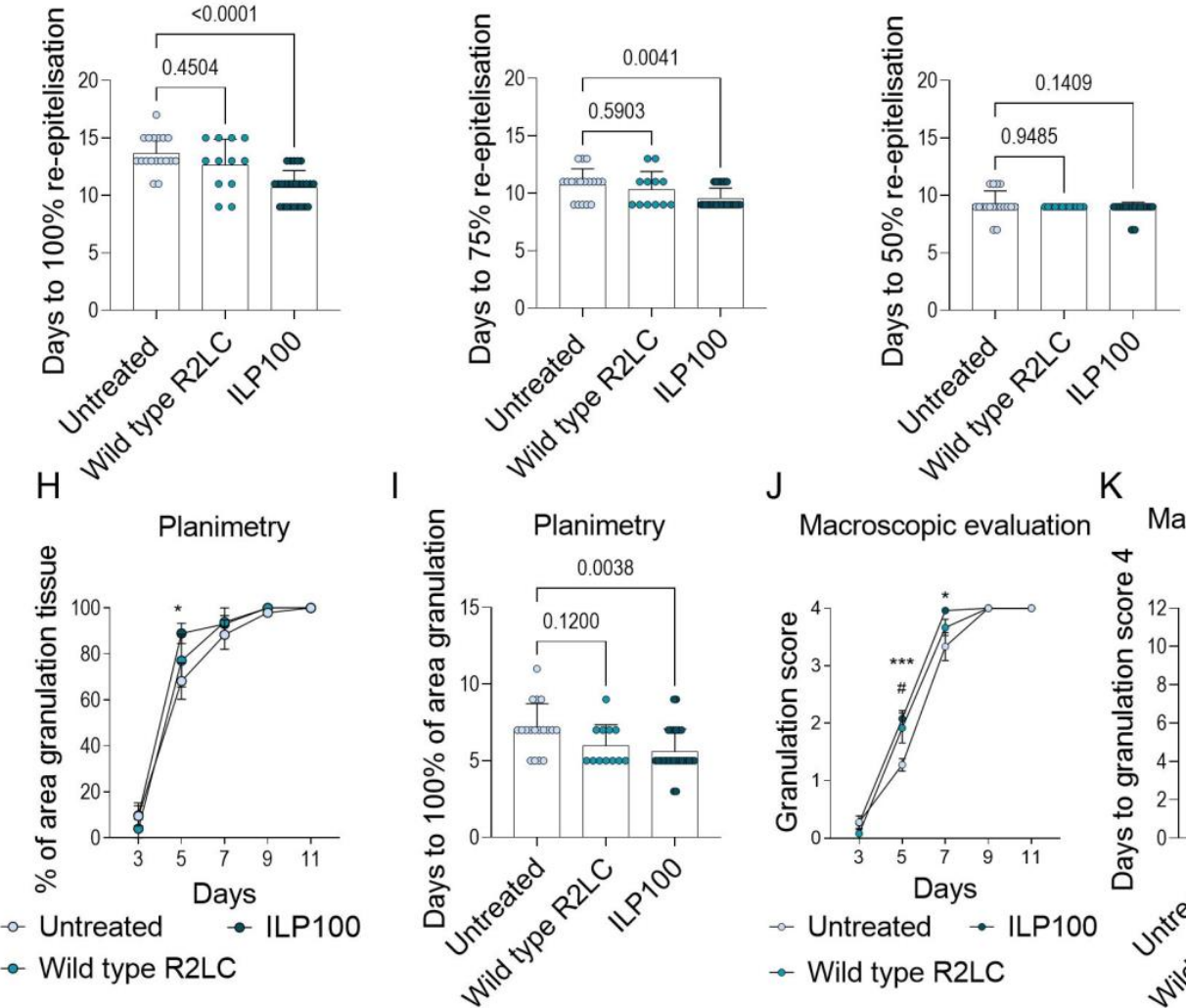

K

Macroscopic evaluation

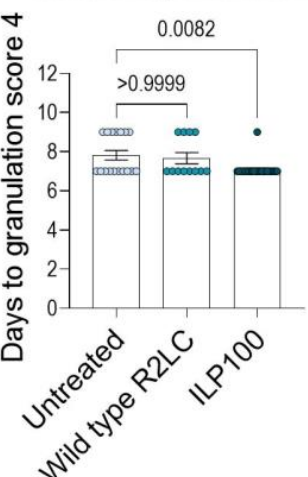

Figure 3. Wound healing assessed by planimetry and macroscopic evaluation in cohort A. (A) shows representative photographs of healing over time where the inserted numbers depict days post-induction and the scale bar corresponds to $1 \mathrm{~cm}$. Wound healing was assessed by planimetry detecting wound area (B), \% of the wound being re-epithelized (C), and area of newly formed epithelia over time (D). Days to $100 \%$ (E), 75\% (F), and 50\% (G) re-epithelization of the wounds were also assessed. In addition, granulation tissue formation was measured as wound area covered by granulation tissue $(\mathbf{H})$ and days to $100 \%$ of the wound area being covered by granulation tissue (I) using planimetry, as well as macroscopically as granulation score (0-4) day 3 to $11(\mathrm{~J})$ and days to 
granulation score $4(\mathbf{K})$. The animals and wounds were divided into the following groups: Untreated $(n=3, N=18)$, Wild type R2LC $(n=3, N=12)$, ILP100 $(n=5, N=26)$. Statistic comparisons between groups were made using Kruskal-Wallis test with Dunn's multiple comparison test (B-K). For (B$\mathbf{D}, \mathbf{H}, \mathbf{J})$ one test was performed for each time point. ${ }^{*}$ Indicates the difference between untreated and ILP100 and \# difference between untreated and wild type R2LC. $\left({ }^{*} p<0.05,{ }^{* *} p<0.01,{ }^{* * *} p<0.001\right)$.

A Macroscopic evaluation

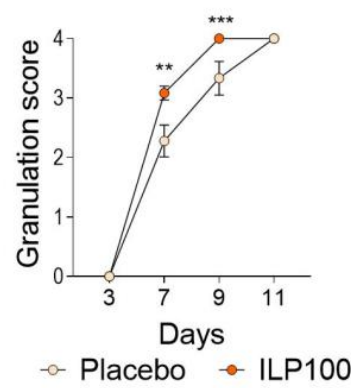

B

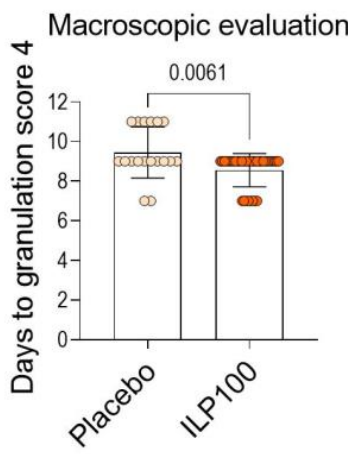

C

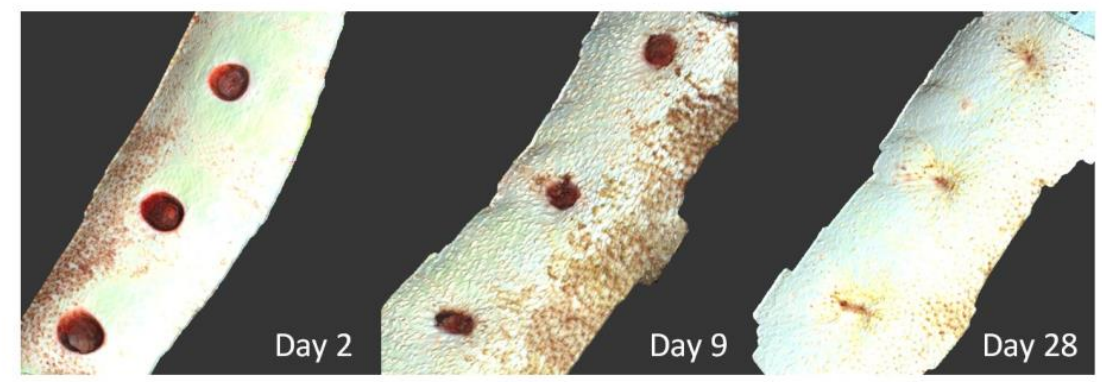

D 3D scans

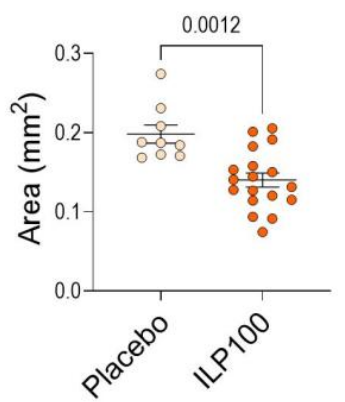

Day 9

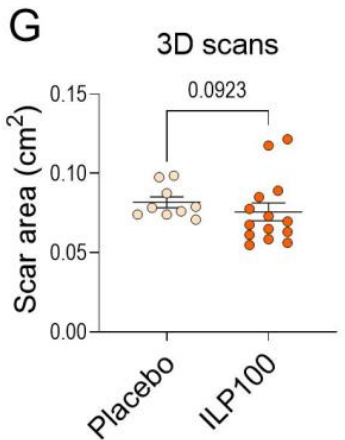

Day 28
E

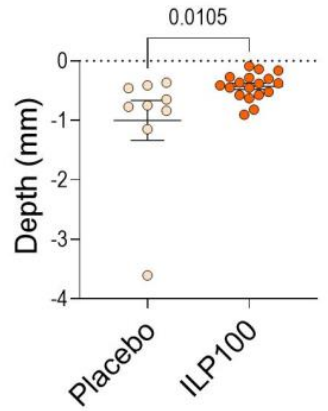

Day 9

$\mathrm{H}$

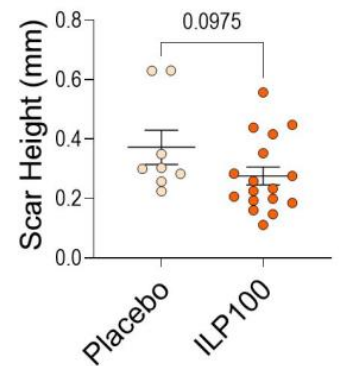

Day 28
F 3D scans

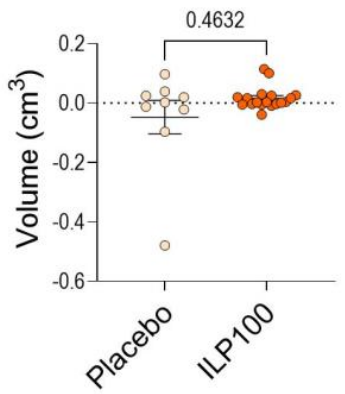

Day 9

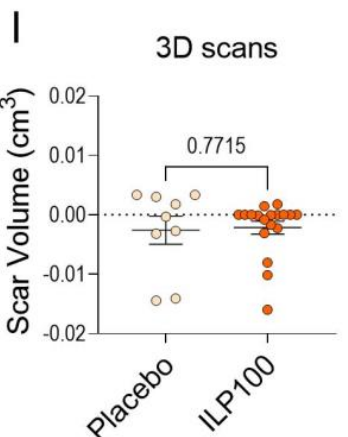

Day 28

Figure 4. Wound healing assessed by macroscopic evaluation and from 3D scans in Cohort B. Wounds 
in Cohort B were macroscopically evaluated for granulation tissue formation by granulation score day 3 to 11 (A) and days to granulation score $4 .{ }^{* *} p<0.01,{ }^{* * *} p<0.001$ (B) (Placebo: $n=3, N=18$, ILP100: $n=6, N=36$ ). The healing was also assessed using $3 \mathrm{D}$ scans, where panel (C) shows representative projections of 3D scans from day 3, day 9, and day 28. From the 3D scans, wound area, wound depth, and wound volume were measured (D-F) for placebo and ILP100-treated wounds day 9 (Placebo: $n=3, N=9$, ILP100: $n=6, N=18$ ). On day 28 when the wounds were healed, scar area, scar height, and scar volume (G-I) were measured from 3D scans, (Placebo: $n=3, N=9$, ILP100: $n=5-6, N=14-18)$. Statistic comparison between the groups were made using Mann-Whitney tests $(\mathbf{A}, \mathbf{B}, \mathbf{D}-\mathbf{I})$.

\subsection{Safety Assessments}

Safety was assessed for all treatments, and there were no reported observations that could be linked to treatment in conventional hematology-, clinical chemistry-, or urine analysis in any sample. In addition, no histopathological findings (for examined samples see Table A1) were reported by the respective pathologist, and the healing of all wounds was categorized as advanced.

Local tolerability was assessed by scoring inflammation of wound edges and surrounding skin. Overall, there were no differences detected between the treatments over time, though a slight increase in inflammation of the surrounding skin was observed in ILP100 treated wounds at day 29 in Cohort A, with an increase from a $0.0( \pm 0.0)$ score in untreated wounds to $0.6( \pm 1.0)$ (Table A2).

Small differences were observed in the hemorrhage scoring. In Cohort A, hemorrhage scoring was slightly increased for wild type- and ILP100-treated wounds compared to untreated wounds at day 7 (untreated: $0.3 \pm 0.6$, representing non- to minimal bleeding, wild type R2LC; $1.4 \pm 0.5$, and ILP100: $1.2 \pm 0.6$, representing minimal to slight bleeding, Table A3). Increased bleeding was also observed on day 7 in Cohort B, with an average hemorrhage score of $0.4 \pm 0.5$ for placebo-, and $0.9 \pm 0.5$ for ILP100-treated wounds. In contrast, decreased bleeding was observed for treated wounds at day 9 in Cohort A and at day 3 and 11 in Cohort B (Table A3). In Cohort A, less exudate was observed on days 2-5 in wild type R2LC- and ILP100-treated wounds compared to untreated wounds (Table A4), but this was not observed in Cohort B. Furthermore, no elevated hypergranulation scoring was observed for the ILP100-treated wounds in either cohort, while wild type R2LC-treated wounds received a slightly increased score compared to untreated wounds in Cohort A (Table A5).

Thus, the safety assessment concluded that topical treatment with ILP100 to induced full-thickness wounds in minipigs was both safe and well-tolerated.

\section{Discussion}

Despite being a significant societal burden in industrialized countries, available treatment options for non-healing wounds are today very limited. This study investigates the biological effects of the drug candidate ILP100 on induced wounds in minipigs by evaluating different and novel methods for the assessment of wound healing. Of the methods evaluated, we found that planimetry reported reduced wound areas (day 9 and 11) and faster healing when compared to 2D photographs, which in turn reported smaller wound areas than 3D scans. Wounds treated with ILP100 demonstrated accelerated healing by advanced re-epithelialization, as revealed by planimetry, 2D photographs, and 3D scans, in addition to higher granulation scores and increased area of granulation, as measured by planimetry.

For successful translation of preclinical projects, the clinical relevance of the models used is essential. The most widely used experimental animals are inbred mice due to their small size, as well as the wide palette of available genetically modified strains. However, rodent skin contains a muscle layer (panniculus carnosus) that enables the contraction of wounds, which does not exist in human skin and complicates translation. In contrast, pig skin not only lacks the contractile muscle layer but also resembles human skin with its 
sparse haircoat, firm attachment to underlying connective tissue, and epidermal turnover time $[11,18,19]$. When the translational success was evaluated in 25 wound healing studies, the agreement between the pre-clinical and clinical outcome was higher for pre-clinical evaluation in pigs $(78 \%)$ than in smaller mammals (53\%) or using in vitro studies (57\%) [20]. In the current study, our previous observation of accelerated wound healing in mice treated with CXCL12-producing L. reuteri R2LC was confirmed to also occur in minipigs, even though with different kinetics.

In addition to inter-species differences in skin anatomy, the complexity of the wound healing process, and particularly that of non-healing wounds and associated underlying pathologies, adds another challenge to the development of new efficient therapies. Indeed, during the last decades, many treatments that passed the safety requirements of Phase I clinical trials later fail to show consistent efficacy in wound healing in Phase II or III [21]. This is likely to depend on several factors, such as differences in the wound healing process between wound types, as well as difficulties in ensuring bioavailability following topical administration due to the high levels of degrading enzymes in the wound environment. Another factor for the limited success of clinical trials is that the only accepted primary endpoint to date is complete wound healing, reported as the time to heal, or the fraction of healed wounds at a relevant time point [16]. However, healing of wounds not only involves a reduction of the wound area through re-epithelialization, but also requires regeneration of tissue in the wound cavity, namely the formation of granulation tissue. In fact, the absence of healthy granulation tissue is a characteristic of non-healing wounds [3,22]. For this reason, solely evaluating wound healing by repeated measurements of wound area does not readily account for the wound healing process. In addition, the depth of the wound has been shown to be a predictor of its healing rate [23], as well as being associated with the risk of amputation in diabetic foot ulcers [24,25]. Therefore, assessment of the granulation tissue and depth measurements are part of many of the assessment tools that have been developed for non-healing wounds, such as Pressure Ulcer Scale for Healing (PUSH), Sussman Wound Healing Tool (SWHT), and Bates-Jensen wound assessment tool (BWAT) [23,26-29]. Of these tools, only BWAT considers the amount of granulation tissue while the others only assess the presence or absence of healthy granulation tissue. Other factors such as exudation, inflammation, and the presence of necrotic tissue or slough may also give an indication on how the healing is progressing [30-32]. Thus, extensive efforts have been made to identify appropriate new primary endpoints for wound healing studies $[15,16,33]$. Even though the primary endpoint of complete healing remains, the FDA recently announced that it is open to discussing new primary endpoints, including (1) Percentage area reduction (PAR), (2) Reduced infection, (3) Reduced pain/reduced analgesia usage, (4) Increased physical function and ambulation, and (5) Quality of life [14].

Translation of preclinical wound therapies is also limited by the fact that there is no current gold standard for evaluating wound healing. For clinical practice, several different wound healing assessment scales have been developed, of which the two more commonly used are the Pressure Ulcer Scale for Healing (PUSH) and Bates-Jensen wound assessment tool (BWAT) [23,26-28]. As mentioned above, these scales score the wounds based on several parameters, including area, depth, and tissue type. However, the dimensions used comprise linear measurements, where the surface area is calculated by measuring the length and the width of the wound (area = length $\times$ width), while wound depth is based on one or a few measurements by a ruler. While these linear measurements are easy to execute, the calculated areas have been shown to overestimate the wound area by up to $40 \%$ [34]. More precise measurements can be achieved by measuring the area within the wound boundaries, as in this study for planimetry, 2D photographs, and 3D scans. Planimetry and wound area measurements from 2D photographs have been demonstrated to have good interand intra-investigator reliability [34-36]. However, while planimetry and 2D photographs reported similar wound areas for most time points in the current study, smaller wounds were detected on days 1 and 2 using 2D photographs compared to planimetry, while the opposite was found on days 9 and 11. The difference observed days 1-2 is in agreement 
with a previous study comparing the same type of methods where mean wound areas directly after induction $\left(0.10-2.20 \mathrm{~cm}^{2}\right)$ were smaller when measured using $2 \mathrm{D}$ photographs compared to planimetry [35]. The differences in measured wound area detected on days 9 and 11 coincide with when newly formed epithelia can first be detected using planimetry. This observation demonstrates an inherent problem with wound healing assessments, namely the definition of the wound boundary. If the wound boundary is delineated by the newly formed epithelia, only methods enabling epithelial detection will identify this new edge, and thereby report smaller wound areas. While the newly formed epithelia are easily identified using conventional histopathology that does not allow studies over time, it requires high-resolution images for repeated evaluations. The limited resolution of the $2 \mathrm{D}$ photographs is probably causing the observed discrepancies in wound areas when assessed by planimetry or 2D photography at the time of formation of thin epithelial layers (day 9-11), even though the individual definition of wound boundaries by the evaluators cannot be excluded. Poor resolution of 2D photographs was a limiting factor when wound diameters obtained from 2D photographs or ultrasound images were compared, as the latter technique resulted in significantly shorter diameters.

However, when designing the protocol of a clinical trial assessing wound healing, it is not only the accuracy of the method, but also several other factors relevant to wound healing assessment, that need to be considered. For instance, 2D photographs offer traceability and a reduced risk of infection, as contact with the wounded area is not required. In addition, neither planimetry nor 2D photographs generate wound depth or volume, which traditionally have required more invasive methods, including a cotton-tipped applicator for the former or filling the wound cavity with saline or alginate gel for the latter [23]. To circumvent the risk of causing infections, 3D non-invasive wound measuring techniques have been developed, but have been limited by costly equipment requiring special training [36]. In recent years, more user-friendly systems have been launched, some of which have been shown to produce reliable area measurements [37-39], while depth and volume measurements are underestimated in some evaluations [37,38]. Here, the Cherry Imaging system comprising a stereoscopic 3D scanner and associated TraceTM software was assessed for healing of full-thickness wounds in minipigs. This system has not previously been used for wounds but has been evaluated in scar models and in patients with hypertrophic and atrophic scars [40], where it demonstrated good intra- and inter-observer repeatability with no difference detected for depressed or elevated scars [40]. When applying this system on induced wounds, we found that 3D scans resulted in larger areas when compared to areas obtained from 2D photographs. This difference could be due to the fact that only the 3D scans take the curvature of the pigs' backs into consideration. A study comparing the areas of human wounds with various sizes $\left(0.3-36.1 \mathrm{~cm}^{2}\right)$ and etiology measured either by 3D images captured with another stereoscopic imaging device or 2D photographs analyzed using ImageJ, did not find any differences between methods [39]. The large range of wound size and the heterogeneity of the assessed wounds might also have contributed to a difference not being detected in this study. In addition, the same study reports that volume measurements acquired from the 3D images correlated well with volume measured by gel application [39].

Immune cells are known to contribute to the distinct phases of the wound healing cascade by different means [41,42]. While innate bactericidal functions are crucial in the early stages following wounding, the tissue restorative functions of predominantly macrophages drive the healing and remodelling processes during the later phases. The healing process is orchestrated by a cascade of growth factors, chemokines, and cytokines, and delivery of these to the wound site has been explored as treatment options for wound healing. Becaplerim, a gel containing recombinant platelet-derived growth factor (PDGF), was the first example of this approach and was approved by the FDA in 1997. Other available treatments that utilize growth factors and chemokines are Platelet-rich plasma (PRP) and amniotic membrane allografts (reviewed in [6]). PRP contains the plasma fraction with high concentrations of platelets and thereby increases the amount of growth factors 
such as TGF- $\beta$, PDGF, and epidermal growth factor (EGF) in the wound following release from platelets $[43,44]$. Amniotic membranes allografts have in multiple studies been shown to have a clinical effect on non-healing wounds, and there are some commercially available options (EpiFix, MiMedx Group, and the Grafix, Osiris Therapeutics) [45]. The amniotic membrane contains a large amount of growth factors, chemokines, and cytokines, including CXCL12 [46]. To utilize the inherent healing properties of wound macrophages, we recently developed an immunotherapeutic approach to kick-start wound healing. Thus, accelerated wound healing of full-thickness wounds was detected in mice following treatment with murine CXCL12-producing L. reuteri R2LC. The accelerated wound healing was most obvious in the days following wound induction, as the area of treated wounds was reduced by $50 \%$ compared to untreated or wild-type L. reuteri treated wounds [8]. In addition, the wounds treated with murine CXCL12-producing L. reuteri R2LC healed on average almost 2 days faster. As a major treatment effect in the mice was seen in the early phase of wound healing, contraction of the wound could be contributing to the observed strong effects, reinforcing the need to test the same therapy in minipigs. Indeed, in the current study in minipigs, the initial reduction of wound size is not seen, even though treatment with ILP100 (freeze-dried formulation of human CXCL12-producing L. reuteri R2LC) still reduces the number of days to complete re-epithelialization. Here, the effect on shortened time to wound healing was demonstrated in two separate cohorts, as Cohort A reports 3 days' faster complete re-epithelialization using planimetry, while Cohort B demonstrates a reduced number of days to $50 \%$ and $75 \%$ re-epithelialization measured from 2D photographs. In fact, the human variant CXCL12 has previously been shown to have a biological effect on wound healing in Yorkshire pigs where healing was accelerated in full-thickness incision wounds treated with scaffolds soaked in human CXCL12 protein or plasmid DNA coding for CXCL12 [47].

In the current studies, both the presence of granulation tissue and the proportion of the wound cavity filled with granulation tissue were scored, with a similar scoring system to BWAT. In addition, the area of the wound covered with granulation tissue was measured by planimetry. We found that wounds treated with ILP100 demonstrated an accelerated formation of granulation tissue, shown by increased granulation scoring on day 5 and day 7 (Cohort A) and day 7 and day 9 (Cohort B), as well as increased area covered with granulation tissue on day 5 (Cohort $\mathrm{A}$ ). Both studies revealed a reduced number of days to reach a granulation score of 4 , indicating that the whole cavity is filled with granulation tissue more quickly. During wound healing, TGF- $\beta$ is one of the major growth factors stimulating the formation of granulation tissue, and is released by wound macrophages and following degradation of extracellular matrix (ECM) [48,49]. Even though macroscopic evaluation of the wound provides important information on the healing process, and there are several validated tools for this as mentioned above, these evaluations are not traceable. Using the Cherry Imaging system, the healing of the cavity may be evaluated objectively by measurement of the depth and volume as well as assessment of the scar height and volume. In accordance with the granulation scoring, the 3D scans revealed reduced depth of wounds at day 9 following treatment with ILP100 when compared to the placebo.

One limitation of the current experimental design is that the two cohorts were studied 2 years apart, which might have resulted in a slight shift in the grading criteria of the granulation tissue. Further, Cohort A included only male pigs whereas Cohort B was conducted in only females. However, all animals in the respective cohort were included in the study within a week and housed in the same stables, limiting the environmental differences and allowing for intra-cohort comparisons. Despite some observed differences in wound healing between the two cohorts following no treatment or treatment with placebo or wild-type bacteria, similar results were observed for treatment efficacy of ILP100. Thus, ILP100-treated wounds in two separate cohorts demonstrated the accelerated formation of granulation tissue and re-epithelialization, signifying the translational potential of our previous observations in mice. 


\section{Conclusions}

We found that topical treatment with the new drug candidate ILP100 to full-thickness wounds in minipigs accelerates healing and is well tolerated. The current study also reveals the need for standardized methods to assess wound healing since differences between methods can be substantial. In addition to educating evaluators to use the same criteria for wound assessment, careful consideration should be taken when choosing methods, including the need for high accuracy, mode of action of the drug candidate, user-friendliness, traceability, and costs, as well as the risk of infection or other disturbances to the wound healing process.

\section{Patents}

ILP100 for the treatment of wounds is protected by patents in multiple countries.

Author Contributions: Conceptualization, P.F., S.R., E.V. and M.P.; formal analysis, E.Ö.; funding acquisition, E.V.; methodology, E.Ö., H.L.T. and P.F.; project administration, E.V.; supervision, S.R. and M.P.; validation, E.Ö. and H.L.T.; visualization, E.Ö.; writing—original draft, E.Ö.; writing—review \& editing, H.L.T., P.F., S.R., E.V. and M.P. All authors have read and agreed to the published version of the manuscript.

Funding: Ilya Pharma received grants from VINNOVA (grant no. 2017-03573), the European Institute of Innovation and Technology (EIT) Health (2016), H2020 SME Instrument phase II under Grant Agreement No 804438 for activities included in this scientific publication. The studies was also supported by Diabetes Wellness (grant to EV), and by the Swedish Research Council (grant no. 2018-02552_VR) and the Knut and Alice Wallenberg foundation through grants to MP.

Institutional Review Board Statement: All experiments were approved by The Danish Veterinary and Food administration Council (Ethical permit number; 2015-15-0201-00713).

Informed Consent Statement: Not applicable.

Data Availability Statement: The data presented in this study are available on request from the corresponding author.

Acknowledgments: We would like to thank Per Sjöberg for their advice relating to study design and regulatory compliance, Pia Dahg for study monitoring, and Tora Frank for her contribution to the 3D scan analysis.

Conflicts of Interest: Ilya Pharma AB is a for-profit SME developing next generation immunotherapies to treat wounds in skin and mucosa. E.V., M.P. and S.R. are co-founders of Ilya Pharma, and MP and SR support the company as experts. E.Ö., H.L. and P.F. are employees at Ilya Pharma.

\section{Appendix A}

Table A1. Organs and tissues examined by histopathology in cohort A and B. * Indicate unilateral sampling and ${ }^{* *}$ bilateral sampling.

Organs and Tissue for Histopathology

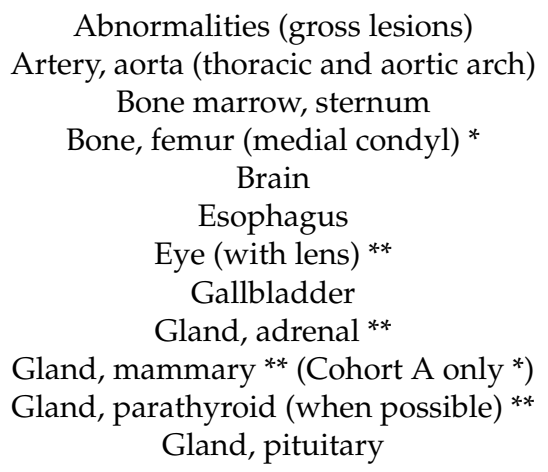

Muscle, skeletal *

Nerve, optic **

Nerve, sciatic * Pancreas

Skin, untreated

Small intestine, duodenum

Small intestine, jejunum

Small intestine, ileum

Spinal cord, thoracic

Spinal cord, lumbar Spleen

Stomach 
Table A1. Cont.

\begin{tabular}{|c|c|}
\hline \multicolumn{2}{|c|}{ Organs and Tissue for Histopathology } \\
\hline Gland, salivary, parotid * & Thymus \\
\hline Gland, salivary, sublingual * & Tongue \\
\hline Gland, salivary, submandibular * & Ureter ** \\
\hline Gland, thyroid & Urinary bladder \\
\hline Heart & Wounds \\
\hline \multicolumn{2}{|l|}{ Joint, femorotibial * } \\
\hline Kidney ${ }^{* *}$ & Cohort A \\
\hline Large intestine, cecum & Gland, prostate \\
\hline Large intestine, colon & Testis \\
\hline \multicolumn{2}{|l|}{ Large intestine, rectum } \\
\hline Larynx & Cohort B \\
\hline Liver (all main lobes) & Lymph node, axillary \\
\hline Lung (cranial and caudal lobes, both sides) & Ovary ** \\
\hline Lymph node, mandibular * & Uterus (horn ${ }^{* *}$, cervix and oviduct ${ }^{* *}$ ) \\
\hline Lymph node, mesenteric & Vagina \\
\hline
\end{tabular}

A

2D photographs
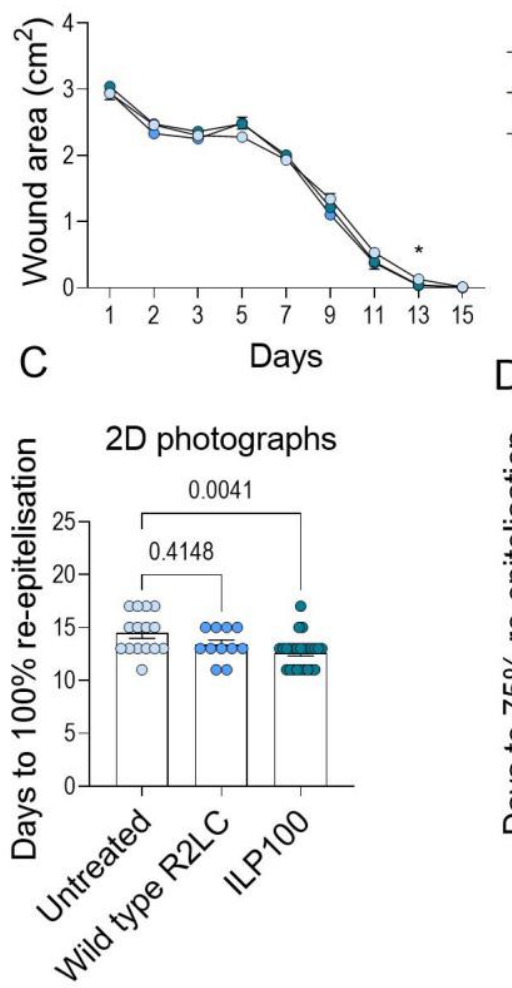

B
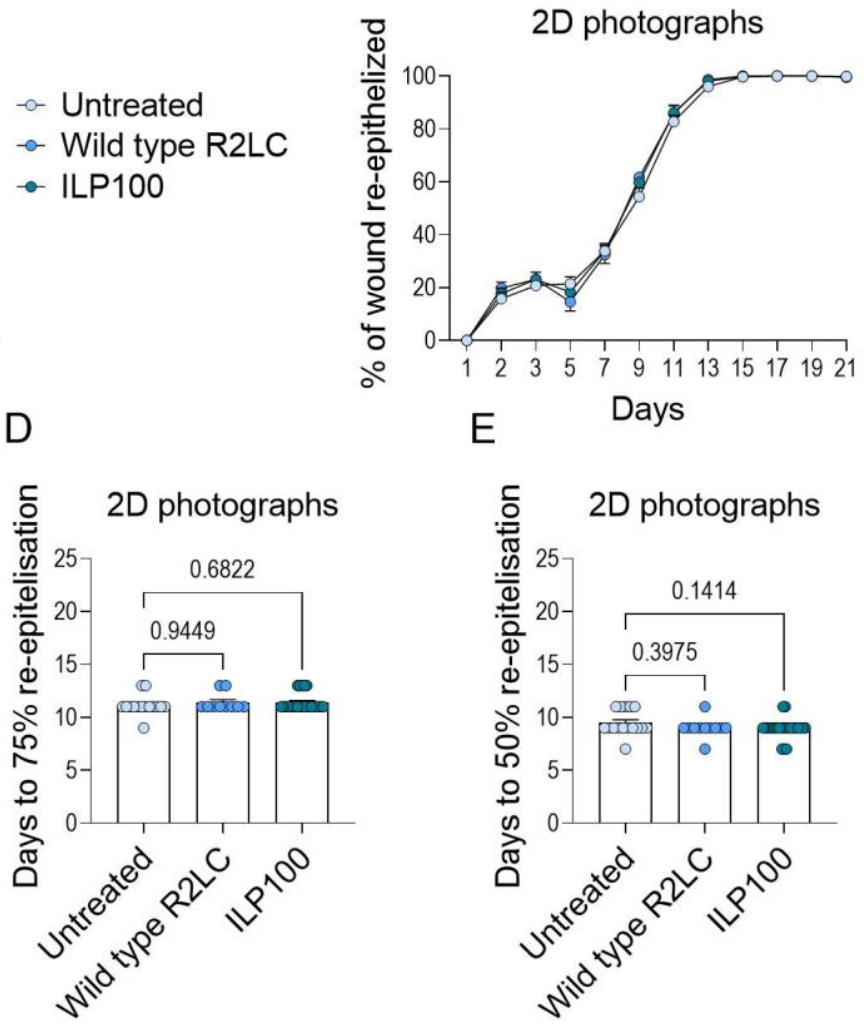

E

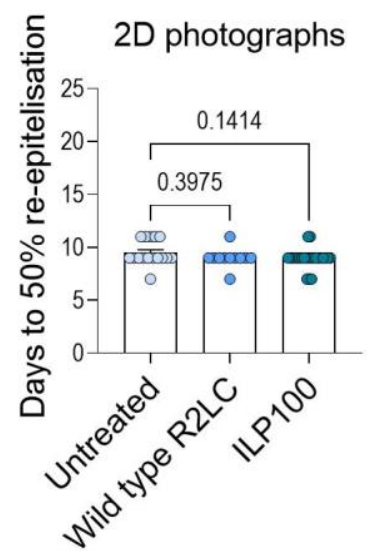

Figure A1. Wound healing assessed from 2D photographs for Cohort A. Wound area and wound healing over time were measured from 2D photographs in Cohort A (A,B), and days to $100 \%(\mathbf{C}), 75 \%$ (D), and 50\% (E) re-epithelization were assessed. (A,B): Untreated $(n=3, N=18)$, Wild type R2LC $(n=3, N=12)$, ILP100 $(n=5, N=26)$. (C-E): Untreated $(n=3, N=18)$, Wild type R2LC $(n=3, N=11)$, ILP100 $(n=5, N=25)$. Kruskal-Wallis test with Dunnett's multiple comparison test $(\mathbf{A}-\mathbf{E}) .{ }^{*} p<0.05$. 
A
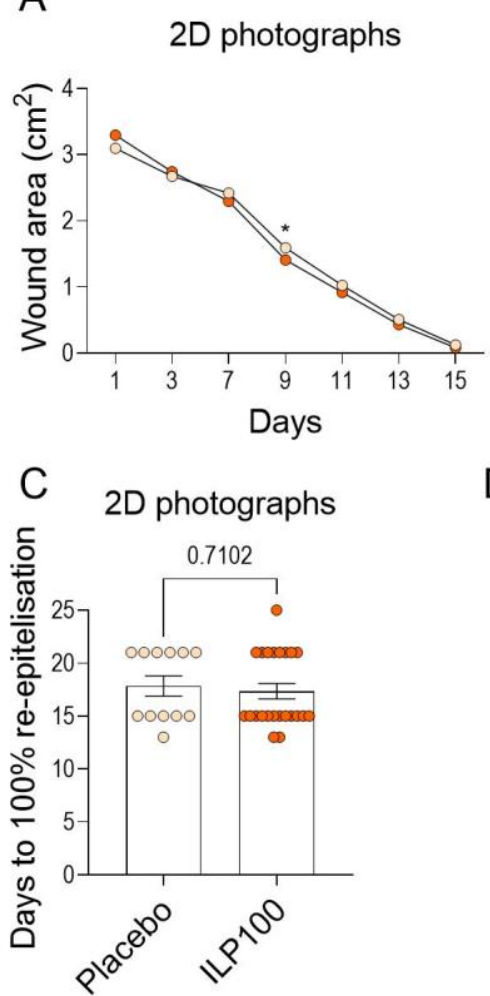

B

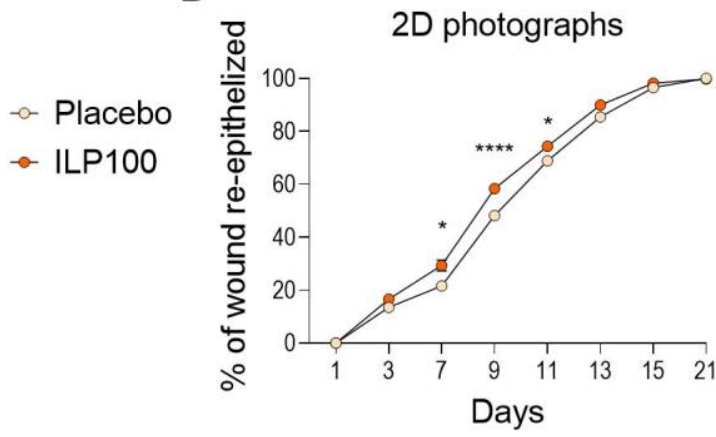

D

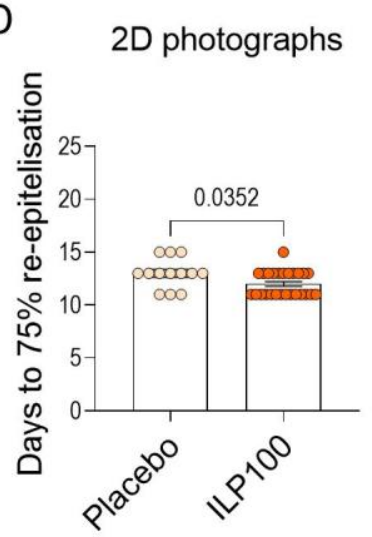

E

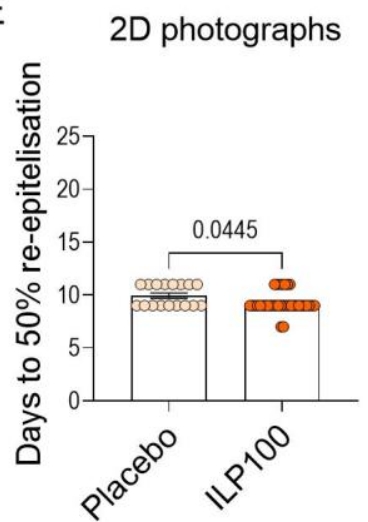

Figure A2. Wound healing assessed from 2D photographs for Cohort B. Wound area and wound healing over time measured from 2D photographs in Cohort B (A,B). From these measurements, days to $100 \%(\mathbf{C}), 75 \%(\mathbf{D})$, and $50 \%$ (E) re-epithelization were assessed. (A,B); Placebo $(n=3, N=18)$, ILP100 $(n=6, N=36)$. (C-E): Placebo $(n=3, N=12-17)$, ILP100 $(n=6, N=23-35)$. Mann-Whitney (A-E). ${ }^{*} p<0.05,{ }^{* * * *} p<0.0001$.

Table A2. Average score (0-4) for wound edge inflammation and inflammation in the surrounding skin for untreated wounds, wounds treated with wild type L. reuteri R2LC or ILP100 in Cohort A and wounds treated with placebo or ILP100 wounds in Cohort B. For time points not included in the table all wounds in all groups were scored as 0 .

\begin{tabular}{|c|c|c|c|c|c|}
\hline \multicolumn{6}{|c|}{ Wound Edge Inflammation (Mean \pm SEM) } \\
\hline Cohort A & Day 3 & Day 5 & Day 7 & Day 9 & $n ; N$ \\
\hline Untreated & $0.17( \pm 0.09)$ & $0.00( \pm 0.00)$ & $0.17( \pm 0.09)$ & $0.00( \pm 0.00)$ & $3 ; 18$ \\
\hline Wild type R2LC & $0.33( \pm 0.14)$ & $0.00( \pm 0.00)$ & $0.00( \pm 0.00)$ & $0.00( \pm 0.00)$ & $3 ; 12$ \\
\hline ILP100 & $0.40( \pm 0.09)$ & $0.17( \pm 0.69)$ & $0.13( \pm 0.8)$ & $0.14( \pm 0.07)$ & $6 ; 30$ \\
\hline Cohort B & Day 3 & - & Day 7 & Day 9 & $n ; N$ \\
\hline Placebo & $0.11( \pm 0.08)$ & - & $0.00( \pm 0.00)$ & $0.00( \pm 0.00)$ & $3 ; 18$ \\
\hline ILP100 & $0.19( \pm 0.07)$ & - & $0.06( \pm 0.04)$ & $0.00( \pm 0.00)$ & $6 ; 36$ \\
\hline \multicolumn{6}{|c|}{ Surrounding Skin Inflammation (Mean \pm SEM) } \\
\hline Cohort A & Day 3 & Day 15 & Day 21 & Day 29 & $n ; N$ \\
\hline Untreated & $0.11( \pm 0.08)$ & $0.00( \pm 0.00)$ & $0.00( \pm 0.00)$ & $0.00( \pm 0.00)$ & $3 ; 18$ \\
\hline Wild type R2LC & $0.25( \pm 0.45)$ & $0.00( \pm 0.00)$ & $0.25( \pm 0.45)$ & $0.58( \pm 0.29)$ & $3 ; 12$ \\
\hline ILP100 & $0.03( \pm 0.03)$ & $0.00( \pm 0.00)$ & $0.13( \pm 0.06)$ & $0.57( \pm 0.18) *$ & $6 ; 30$ \\
\hline Cohort B & Day 3 & Day 15 & Day 21 & Day 28 & $n ; N$ \\
\hline Placebo & $0.00( \pm 0.00)$ & $0.22( \pm 0.10)$ & $0.17( \pm 0.09)$ & $0.11( \pm 0.08)$ & $3 ; 18$ \\
\hline ILP100 & $0.00( \pm 0.00)$ & $0.00( \pm 0.00) *$ & $0.00( \pm 0.00)^{*}$ & $0.00( \pm 0.00)$ & $6 ; 36$ \\
\hline
\end{tabular}


Table A3. Average score (0-4) for hemorrhage for untreated wounds, wounds treated with wild type L. reuteri R2LC or ILP100 in Cohort A and wounds treated with placebo or ILP100 wounds in Cohort B. For time points not included in the table, all wounds in all groups were scored as $0 .{ }^{*} p<0.05$.

\begin{tabular}{|c|c|c|c|c|c|c|c|c|}
\hline \multicolumn{9}{|c|}{ Hemorrhage (Mean \pm SEM) } \\
\hline Cohort A & Day 2 & Day 3 & Day 5 & Day 7 & Day 9 & Day 11 & Day 13 & $n ; N$ \\
\hline Untreated & $0.50( \pm 0.62)$ & $0.22( \pm 0.10)$ & $0.33( \pm 0.11)$ & $0.28( \pm 0.14)$ & $0.89( \pm 0.08)$ & $0.11( \pm 0.08)$ & $0.03( \pm 0.03)$ & $3 ; 18$ \\
\hline $\begin{array}{l}\text { Wild type } \\
\text { R2LC }\end{array}$ & $0.75( \pm 0.22)$ & $0.08( \pm 0.08)$ & $0.33( \pm 0.14)$ & $1.42( \pm 0.15) *$ & $0.83( \pm 0.17)$ & $0.00( \pm 0.00)$ & $0.00( \pm 0.00)$ & $3 ; 12$ \\
\hline ILP100 & $0.17( \pm 0.07)$ & $0.10( \pm 0.06)$ & $0.23( \pm 0.08)$ & $1.17( \pm 0.11) *$ & $0.30( \pm 0.09) *$ & $0.00( \pm 0.00)$ & $0.00( \pm 0.00)$ & $6 ; 30$ \\
\hline Cohort B & - & Day 3 & - & Day 7 & Day 9 & Day 11 & Day 13 & $n ; N$ \\
\hline Placebo & - & $1.17( \pm 0.12)$ & - & $0.39( \pm 0.12)$ & $0.56( \pm 0.12)$ & $0.61( \pm 0.12)$ & $0.11( \pm 0.08)$ & $3 ; 18$ \\
\hline ILP100 & - & $0.47( \pm 0.13) *$ & - & $0.94( \pm 0.09) *$ & $0.47( \pm 0.08)$ & $0.22( \pm 0.07) *$ & $0.06( \pm 0.04)$ & $6 ; 36$ \\
\hline
\end{tabular}

Table A4. Average score (0-4) for exudate for untreated wounds, wounds treated with wild type $L$. reuteri $\mathrm{R} 2 \mathrm{LC}$ or ILP100 in Cohort A and wounds treated with placebo or ILP100 wounds in Cohort B. For time points not included in the table, all wounds in all groups were scored as $0 .{ }^{*} p<0.05$.

\begin{tabular}{|c|c|c|c|c|c|c|c|c|c|}
\hline \multicolumn{10}{|c|}{ Exudate (Mean \pm SEM) } \\
\hline $\begin{array}{l}\text { Cohort A } \\
\text { Untreated }\end{array}$ & $\begin{array}{c}\text { Day 2 } \\
1.28( \pm 0.11)\end{array}$ & $\begin{array}{c}\text { Day 3 } \\
1.39( \pm 0.16)\end{array}$ & $\begin{array}{c}\text { Day 5 } \\
1.72( \pm 0.11)\end{array}$ & $\begin{array}{c}\text { Day 7 } \\
0.61( \pm 0.12)\end{array}$ & $\begin{array}{c}\text { Day } 9 \\
0.39( \pm 0.14)\end{array}$ & $\begin{array}{c}\text { Day 11 } \\
0.17( \pm 0.09)\end{array}$ & $\begin{array}{c}\text { Day 13 } \\
0.00( \pm 0.00)\end{array}$ & $\begin{array}{c}\text { Day 19 } \\
0.00( \pm 0.00)\end{array}$ & $\begin{array}{l}n ; N \\
3 ; 18\end{array}$ \\
\hline $\begin{array}{l}\text { Wild type } \\
\text { R2LC }\end{array}$ & $0.83( \pm 0.11)^{*}$ & $0.67( \pm 0.14)^{*}$ & $0.92( \pm 0.15) *$ & $0.17( \pm 0.11)$ & $0.25( \pm 0.13)$ & $0.08( \pm 0.08)$ & $0.00( \pm 0.00)$ & $0.08( \pm 0.08)$ & $3 ; 12$ \\
\hline ILP100 & $0.63( \pm 0.09)^{*}$ & $0.67( \pm 0.10) *$ & $0.80( \pm 0.12) *$ & $0.57( \pm 0.11)$ & $0.50( \pm 0.10)$ & $0.03( \pm 0.03)$ & $0.07( \pm 0.05)$ & $0.00( \pm 0.00)$ & $6 ; 30$ \\
\hline Cohort B & - & Day 3 & - & Day 7 & Day 9 & Day 11 & Day 13 & - & $n ; N$ \\
\hline Placebo & - & $1.56( \pm 0.12)$ & - & $1.17( \pm 0.17)$ & $0.56( \pm 0.12)$ & $0.00( \pm 0.00)$ & $0.00( \pm 0.00)$ & - & $3 ; 18$ \\
\hline ILP100 & - & $1.50( \pm 0.08)$ & - & $0.75( \pm 0.11)$ & $0.47( \pm 0.08)$ & $0.17( \pm 0.06)$ & $0.06( \pm 0.04)$ & - & $6 ; 36$ \\
\hline
\end{tabular}

Table A5. Average score (0-4) for hypergranulation for untreated wounds, wounds treated with wild type L. reuteri R2LC or ILP100 in Cohort A and wounds treated with placebo or ILP100 wounds in Cohort B. For time points not included in the table, all wounds in all groups were scored as 0. * $p<0.05$.

\begin{tabular}{ccccc}
\hline \multicolumn{4}{c}{ Hypergranulation (Mean \pm SEM) } \\
\hline Cohort A & Day 7 & Day 9 & Day 11 & $n ; \boldsymbol{N}$ \\
Untreated & $0.00( \pm 0.00)$ & $0.17( \pm 0.09)$ & $0.06( \pm 0.06)$ & $3 ; 18$ \\
Wild type R2LC & $0.25( \pm 0.13)$ & $0.17( \pm 0.11)^{*}$ & $0.00( \pm 0.00)$ & $3 ; 12$ \\
ILP100 & $0.00( \pm 0.00)$ & $0.00( \pm 0.00)$ & $0.00( \pm 0.00)$ & $6 ; 30$ \\
Cohort B & Day 7 & Day 9 & Day 11 & $n ; N$ \\
Placebo & $0.00( \pm 0.00)$ & $0.00( \pm 0.00)$ & $0.33( \pm 0.11)$ & $3 ; 18$ \\
ILP100 & $0.03( \pm 0.03)$ & $0.08( \pm 0.05)$ & $0.22( \pm 0.07)$ & $6 ; 36$ \\
\hline
\end{tabular}

\section{References}

1. Ashcroft, G.S.; Horan, M.A.; Ferguson, M.W. The Effects of Ageing on Cutaneous Wound Healing in Mammals. J. Anat. 1995, 187 Pt 1, 1-26. [PubMed]

2. Demidova-Rice, T.N.; Hamblin, M.R.; Herman, I.M. Acute and Impaired Wound Healing: Pathophysiology and Current Methods for Drug Delivery, Part 1 Normal and Chronic Wounds Biology, Causes, and Approaches to Care. Adv. Ski. Wound Care 2012, 25, 304-314. [CrossRef] [PubMed]

3. Grey, J.E.; Enoch, S.; Harding, K.G. Wound Assessment. BMJ 2006, 332, 285-288. [CrossRef] [PubMed]

4. Chan, B.; Cadarette, S.; Wodchis, W.; Wong, J.; Mittmann, N.; Krahn, M. Cost-of-Illness Studies in Chronic Ulcers: A Systematic Review. J. Wound Care 2017, 26, S4-S14. [CrossRef]

5. Statens beredning för medicinsk utvärdering. Svårläkta sår hos Äldre: Prevention och Behandling: En Systematisk Litteraturöversikt; Statens Beredning för Medicinsk utvärdering (SBU): Stockholm, Sweden, 2014; ISBN 978-91-85413-67-6.

6. Öhnstedt, E.; Lofton Tomenius, H.; Vågesjö, E.; Phillipson, M. The Discovery and Development of Topical Medicines for Wound Healing. Expert Opin. Drug Discov. 2019, 14, 485-497. [CrossRef] [PubMed]

7. Yager, D.R.; Nwomeh, B.C. The Proteolytic Environment of Chronic Wounds. Wound Repair Regen. 1999, 7, 433-441. [CrossRef] [PubMed]

8. Vågesjö, E.; Öhnstedt, E.; Mortier, A.; Lofton, H.; Huss, F.; Proost, P.; Roos, S.; Phillipson, M. Accelerated Wound Healing in Mice by On-Site Production and Delivery of CXCL12 by Transformed Lactic Acid Bacteria. Proc. Natl. Acad. Sci. USA 2018, 115, 1895-1900. [CrossRef]

9. Summerfield, A.; Meurens, F.; Ricklin, M.E. The Immunology of the Porcine Skin and Its Value as a Model for Human Skin. Mol. Immunol. 2015, 66, 14-21. [CrossRef] 
10. Zomer, H.D.; Trentin, A.G. Skin Wound Healing in Humans and Mice: Challenges in Translational Research. J. Dermatol. Sci. 2018, 90, 3-12. [CrossRef]

11. Seaton, M.; Hocking, A.; Gibran, N.S. Porcine Models of Cutaneous Wound Healing. ILAR J. 2015, 56, 127-138. [CrossRef]

12. Zindle, J.K.; Wolinsky, E.; Bogie, K.M. A Review of Animal Models from 2015 to 2020 for Preclinical Chronic Wounds Relevant to Human Health. J. Tissue Viability 2021, 30, 291-300. [CrossRef]

13. U.S. Department of Health and Human Services Food and Drug Administration. Guidance for Industry Chronic Cutaneous Ulcer and Burn Wounds-Developing Products for Treatment; U.S. Department of Health and Human Services Food and Drug Administration: Silver Spring, MA, USA, 2006.

14. Dotson, P. Who Said Persistence Doesn't Pay Off? FDA Closer to New Primary Wound Care Endpoints. Today's Wound Clin. 2020, $14,18-19$.

15. Driver, V.R.; Gould, L.J.; Dotson, P.; Allen, L.L.; Carter, M.J.; Bolton, L.L. Evidence Supporting Wound Care End Points Relevant to Clinical Practice and Patients' Lives. Part 2. Literature Survey: Validation of Wound Care End Points. Wound Repair Regen. 2019, 27, 80-89. [CrossRef]

16. Driver, V.R.; Gould, L.J.; Dotson, P.; Gibbons, G.W.; Li, W.W.; Ennis, W.J.; Kirsner, R.S.; Eaglstein, W.H.; Bolton, L.L.; Carter, M.J. Identification and Content Validation of Wound Therapy Clinical Endpoints Relevant to Clinical Practice and Patient Values for FDA Approval. Part 1. Survey of the Wound Care Community: Identification and Content Validation of Endpoints. Wound Repair Regen. 2017, 25, 454-465. [CrossRef] [PubMed]

17. Salameh, F.; Koren, A.; Sprecher, E.; Artzi, O. Novel Stereoscopic Optical System for Objectively Measuring Above-Surface Scar Volume-First-Time Quantification of Responses to Various Treatment Modalities. Dermatol. Surg. 2018, 44, 848-854. [CrossRef]

18. Mangelsdorf, S.; Vergou, T.; Sterry, W.; Lademann, J.; Patzelt, A. Comparative Study of Hair Follicle Morphology in Eight Mammalian Species and Humans. Ski. Res. Technol. 2014, 20, 147-154. [CrossRef] [PubMed]

19. Otberg, N.; Richter, H.; Schaefer, H.; Blume-Peytavi, U.; Sterry, W.; Lademann, J. Variations of Hair Follicle Size and Distribution in Different Body Sites. J. Investig. Dermatol. 2004, 122, 14-19. [CrossRef]

20. Sullivan, T.P.; Eaglstein, W.H.; Davis, S.C.; Mertz, P. The Pig as a Model for Human Wound Healing. Wound Repair Regen. 2001, 9 , 66-76. [CrossRef]

21. Darwin, E.; Tomic-Canic, M. Healing Chronic Wounds: Current Challenges and Potential Solutions. Curr. Dermatol. Rep. 2018, 7 , 296-302. [CrossRef] [PubMed]

22. Gulf Diabetic Foot Working Group Identification and Management of Infection in Diabetic Foot Ulcers: International Consensus; Wounds international: London, UK, 2017.

23. Sussman, C.; Bates-Jensen, B.M. Wound Care: A Collaborative Practice Manual for Health Professionals; Wolters Kluwer Health/Lippincott Williams \& Wilkins: Philadelphia, PA, USA, 2012; ISBN 978-1-60831-715-8.

24. Jeong, E.-G.; Cho, S.S.; Lee, S.-H.; Lee, K.-M.; Woo, S.-K.; Kang, Y.; Yun, J.-S.; Cha, S.-A.; Kim, Y.-J.; Ahn, Y.-B.; et al. Depth and Combined Infection Is Important Predictor of Lower Extremity Amputations in Hospitalized Diabetic Foot Ulcer Patients. Korean J. Intern. Med. 2018, 33, 952-960. [CrossRef] [PubMed]

25. Choi, E.P.H.; Chin, W.Y.; Wan, E.Y.F.; Lam, C.L.K. Evaluation of the Internal and External Responsiveness of the Pressure Ulcer Scale for Healing ( PUSH ) Tool for Assessing Acute and Chronic Wounds. J. Adv. Nurs. 2016, 72, 1134-1143. [CrossRef] [PubMed]

26. Gardner, S.E.; Frantz, R.A.; Bergquist, S.; Shin, C.D. A Prospective Study of the Pressure Ulcer Scale for Healing (PUSH). J. Gerontol. Ser. A Biol. Sci. Med. Sci. 2005, 60, 93-97. [CrossRef]

27. Stotts, N.A.; Rodeheaver, G.T.; Thomas, D.R.; Frantz, R.A.; Bartolucci, A.A.; Sussman, C.; Ferrell, B.A.; Cuddigan, J.; Maklebust, J. An Instrument to Measure Healing in Pressure Ulcers: Development and Validation of the Pressure Ulcer Scale for Healing (PUSH). J. Gerontol. Ser. A Biol. Sci. Med. Sci. 2001, 56, M795-M799. [CrossRef]

28. Sussman, C.; Swanson, G. Utility of the Sussman Wound Healing Tool in Predicting Wound Healing Outcomes in Physical Therapy. Adv. Wound Care 1997, 10, 74-77.

29. Hasatsri, S.; Aramwit, P. Nontraditional Methods to Evaluate Wound Healing. Dermatol. Surg. 2017, 43, 342-350. [CrossRef]

30. Leaper, D.J.; Schultz, G.; Carville, K.; Fletcher, J.; Swanson, T.; Drake, R. Extending the TIME Concept: What Have We Learned in the Past 10 Years? Int. Wound J. 2012, 9, 1-19. [CrossRef]

31. St-Supery, V.; Tahiri, Y.; Sampalis, J.; Brutus, J.-P.; Harris, P.G.; Nikolis, A. Wound Healing Assessment: Does the Ideal Methodology for a Research Setting Exist? Ann. Plast. Surg. 2011, 67, 193-200. [CrossRef]

32. Gould, L.J.; Liu, J.; Wan, R.; Carter, M.J.; Dotson, M.(P.); Driver, V.R. Evidence Supporting Wound Care End Points Relevant to Clinical Practice and Patients' Lives. Part 3: The Patient Survey. Wound Repair Regen. 2021, 29, 60-69. [CrossRef]

33. Rogers, L.C.; Bevilacqua, N.J.; Armstrong, D.G.; Andros, G. Digital Planimetry Results in More Accurate Wound Measurements: A Comparison to Standard Ruler Measurements. J. Diabetes Sci. Technol. 2010, 4, 799-802. [CrossRef] [PubMed]

34. Thawer, H.A.; Houghton, P.E.; Woodbury, M.G.; Keast, D.; Campbell, K. A Comparison of Computer-Assisted and Manual Wound Size Measurement. Ostomy Wound Manag. 2002, 48, 46-53.

35. Jørgensen, L.B.; Sørensen, J.A.; Jemec, G.B.; Yderstraede, K.B. Methods to Assess Area and Volume of Wounds-A Systematic Review: Review of Methods to Assess Wound Size. Int. Wound J. 2016, 13, 540-553. [CrossRef]

36. Davis, K.E.; Constantine, F.C.; MacAslan, E.C.; Bills, J.D.; Noble, D.L.; Lavery, L.A. Validation of a Laser-Assisted Wound Measurement Device for Measuring Wound Volume. J. Diabetes Sci. Technol. 2013, 7, 1161-1166. [CrossRef] 
37. Jun, D.; Choi, H.; Kim, J.; Lee, M.; Kim, S.; Jo, D.; Kim, C.; Shin, D. Efficacy of the Mobile Three-Dimensional Wound Measurement System in Pressure Ulcer Assessment. J. Wound Manag. Res. 2019, 15, 78-84. [CrossRef]

38. Jørgensen, L.B.; Skov-Jeppesen, S.M.; Halekoh, U.; Rasmussen, B.S.; Sørensen, J.A.; Jemec, G.B.E.; Yderstræde, K.B. Validation of Three-dimensional Wound Measurements Using a Novel 3D-WAM Camera. Wound Repair Regen. 2018, 26, 456-462. [CrossRef]

39. Hendel, K.; Ortner, V.K.; Fuchs, C.S.K.; Eckhouse, V.; Haedersdal, M. Dermatologic Scar Assessment With Stereoscopic Imaging and Digital Three-Dimensional Models: A Validation Study. Lasers Surg. Med. 2021, 53, 1043-1049. [CrossRef]

40. Lucas, T.; Waisman, A.; Ranjan, R.; Roes, J.; Krieg, T.; Müller, W.; Roers, A.; Eming, S.A. Differential Roles of Macrophages in Diverse Phases of Skin Repair. J. Immunol. 2010, 184, 3964-3977. [CrossRef] [PubMed]

41. Phillipson, M.; Kubes, P. The Healing Power of Neutrophils. Trends Immunol. 2019, 40, 635-647. [CrossRef]

42. Chicharro-Alcántara, D.; Rubio-Zaragoza, M.; Damiá-Giménez, E.; Carrillo-Poveda, J.; Cuervo-Serrato, B.; Peláez-Gorrea, P.; Sopena-Juncosa, J. Platelet Rich Plasma: New Insights for Cutaneous Wound Healing Management. J. Funct. Biomater. 2018, 9, 10. [CrossRef] [PubMed]

43. Martinez-Zapata, M.J.; Martí-Carvajal, A.J.; Solà, I.; Expósito, J.A.; Bolíbar, I.; Rodríguez, L.; Garcia, J.; Zaror, C. Autologous Platelet-Rich Plasma for Treating Chronic Wounds. Cochrane Database Syst. Rev. 2016. [CrossRef]

44. Lakmal, K.; Basnayake, O.; Hettiarachchi, D. Systematic Review on the Rational Use of Amniotic Membrane Allografts in Diabetic Foot Ulcer Treatment. BMC Surg. 2021, 21, 87. [CrossRef]

45. Cazzell, S.; Vayser, D.; Pham, H.; Walters, J.; Reyzelman, A.; Samsell, B.; Dorsch, K.; Moore, M. A Randomized Clinical Trial of a Human Acellular Dermal Matrix Demonstrated Superior Healing Rates for Chronic Diabetic Foot Ulcers over Conventional Care and an Active Acellular Dermal Matrix Comparator: Clinical Trial of D-ADM Demonstrated Superior Healing Rates for Chronic Diabetic Foot Ulcers. Wound Repair Regen. 2017, 25, 483-497. [CrossRef] [PubMed]

46. Rabbany, S.Y.; Pastore, J.; Yamamoto, M.; Miller, T.; Rafii, S.; Aras, R.; Penn, M. Continuous Delivery of Stromal Cell-Derived Factor-1 from Alginate Scaffolds Accelerates Wound Healing. Cell Transplant. 2010, 19, 399-408. [CrossRef] [PubMed]

47. Xue, M.; Jackson, C.J. Extracellular Matrix Reorganization During Wound Healing and Its Impact on Abnormal Scarring. Adv. Wound Care 2015, 4, 119-136. [CrossRef]

48. Landén, N.X.; Li, D.; Ståhle, M. Transition from Inflammation to Proliferation: A Critical Step during Wound Healing. Cell. Mol. Life Sci. 2016, 73, 3861-3885. [CrossRef]

49. Schultz, G.S.; Wysocki, A. Interactions between Extracellular Matrix and Growth Factors in Wound Healing. Wound Repair Regen. 2009, 17, 153-162. [CrossRef] [PubMed] 